University of Nebraska - Lincoln

DigitalCommons@University of Nebraska - Lincoln

2011

\title{
Effects of Damköhler number on flame extinction and reignition in turbulent non-premixed flames using DNS
}

\author{
David Lignell \\ Brigham Young University, Provo, UT \\ Jacqueline Chen \\ Sandia National Laboratories, jhchen@sandia.gov \\ Hans Schmutz \\ Brigham Young University, Provo, UT
}

Follow this and additional works at: https://digitalcommons.unl.edu/usdoepub

Part of the Bioresource and Agricultural Engineering Commons

Lignell, David; Chen, Jacqueline; and Schmutz, Hans, "Effects of Damköhler number on flame extinction and reignition in turbulent non-premixed flames using DNS" (2011). US Department of Energy

Publications. 123.

https://digitalcommons.unl.edu/usdoepub/123

This Article is brought to you for free and open access by the U.S. Department of Energy at DigitalCommons@University of Nebraska - Lincoln. It has been accepted for inclusion in US Department of Energy Publications by an authorized administrator of DigitalCommons@University of Nebraska - Lincoln. 


\title{
Effects of Damköhler number on flame extinction and reignition in turbulent non-premixed flames using DNS
}

\author{
David O. Lignell $^{\mathrm{a}, *}$, Jacqueline H. Chen ${ }^{\mathrm{b}}$, Hans A. Schmutz ${ }^{\mathrm{a}}$ \\ ${ }^{a}$ Department of Chemical Engineering, Brigham Young University, Provo, UT 84602, United States \\ ${ }^{\mathrm{b}}$ Reacting Flow Research Department, Combustion Research Facility, Sandia National Laboratories, Livermore, CA 94551, United States
}

\section{A R T I C L E I N F O}

Article history:

Received 27 April 2010

Received in revised form 20 June 2010

Accepted 20 October 2010

\section{Keywords:}

DNS

Non-premixed

Extinction

Reignition

Progress variable

\begin{abstract}
A B S T R A C T
Results from a parametric study of flame extinction and reignition with varying Damköhler number using direct numerical simulation are presented. Three planar, non-premixed ethylene jet flames were simulated at a constant Reynolds number of 5120. The fuel and oxidizer stream compositions were varied to adjust the steady laminar extinction scalar dissipation rate, while maintaining constant flow and geometric conditions. Peak flame extinction varies from approximately $40 \%$ to nearly global blowout as the Damköhler number decreases. The degree of extinction significantly affects the development of the jets and the degree of mixing of fuel, oxidizer, and combustion products prior to reignition. The global characteristics of the flames are presented along with an analysis of the modes of reignition. It is found that the initially non-premixed flame undergoing nearly global extinction reignites through premixed flame propagation in a highly stratified mixture. A progress variable is defined and a budget of key terms in its transport equation is presented.
\end{abstract}

(c) 2011 The Combustion Institute. Published by Elsevier Inc. All rights reserved.

\section{Introduction}

Flame extinction and reignition are important processes in non-premixed combustion under highly turbulent conditions. Non-premixed flames occur at the stoichiometric interface between fuel and oxidizer streams, and the rate of combustion is limited by the rate of diffusive mixing of the streams. Turbulence acts to increase flame surface area and scalar gradients, which increases the rate of diffusive mixing. These high mixing rates allow smaller combustion volumes and higher heat release rates. However, as mixing rates increase, finite rate chemical kinetic effects become important, and flame extinction may occur if rates of diffusive heat loss exceed the heat release rate of combustion. Local flame extinction results in flame holes through which unburned fuel may escape, reducing combustion efficiency and allowing fuel emission. High rates of flame extinction may result in unstable combustion, flame liftoff, and if excessive, global flame blowout, posing operational and safety hazards in combustion equipment.

Flame extinction occurs in regions of high turbulent strain, e.g., in the near field of jet flames. As turbulent strain rates decrease, extinguished regions may reignite. The mechanisms and modes of reignition, and the development of turbulent combustion models that can accurately and reliably capture such processes, are the subjects of

* Corresponding author. Address: 350 Clyde Building, Provo, UT 84602, United States. Fax: +1 8014220151

E-mail address: davidlignell@byu.edu (D.O. Lignell). ongoing research. Reignition of extinguished fluid parcels may occur through several possible mechanisms, including:

- Auto-ignition, possibly coupled with ignition front propagation. This mechanism is expected to occur in regions for which the homogeneous ignition delay time is low and temperature gradients are not too large $[1,2]$.

- Edge flame propagation along the (nominally) stoichiometric surface of the extinguished region. By this mechanism a flame hole is essentially healed as burning portions of the stoichiometric surface propagate into extinguished regions under favorable strain [3]. A review of edge flames is given by Buckmaster [4].

- Turbulent flame folding. Reignition may occur through turbulent flame folding, where burning regions or hot combustion products are folded onto extinguished regions, which reignite through diffusive heat and mass transfer $[3,5]$.

- Premixed flame propagation. If the extent of mixing is sufficient during periods of extinction and reignition, a premixed flame may develop and propagate. This flame propagation occurs in a partially premixed mode through a non-homogeneous mixture of varying stoichiometry and temperature, both of which impact the flame speed. Subsequent reaction between rich and lean combustion products may also be important. This mode is expected under conditions of severe and pervasive extinction, but short of flame blowout.

While many experimental investigations of diffusion flames with extinction phenomena have been performed (see e.g., [6], 
references in [3]), the most detailed information available are twodimensional images of the three dimensional flow. Direct numerical simulation (DNS) is currently the only approach that provides detailed temporally and spatially resolved information of the flow field and scalar state space (though at relatively modest Reynolds numbers). Earlier DNS studies have been limited to relatively simple configurations (homogeneous decaying turbulence) and simple chemistry, e.g., [7-9]. Pantano [3] simulated a three-dimensional methane jet flame with a four-step reduced chemical mechanism. Recently, simulations with detailed combustion chemistry have been performed in a methane jet in two dimensions [10]. Hawkes et al. [5,11] performed a parametric study of extinction and reignition in planar, temporally-evolving $\mathrm{CO} / \mathrm{H}_{2}$ jet flames with detailed chemistry and varying Reynolds number. In that study, analysis of isosurfaces of a reactive scalar showed the dominant mode of flame reignition to occur via edge flame propagation and turbulent flame folding [5]. Edge flame propagation was found to dominate in regions of lower scalar dissipation rate, whereas the highest rates of reignition were found to occur by flame folding at higher scalar dissipation rates.

Here, we present DNS results of a parametric study of extinction and reignition in non-premixed, planar, temporally evolving ethylene jet flames. The configuration and flow parameters are chosen to match those of the medium Reynolds number simulation of Hawkes et al. [11]. In this study, rather than vary the Reynolds number at fixed Damköhler number, as in [11], the Damköhler number is varied at fixed Reynolds number. It is expected that the extinction and reignition properties of ethylene are significantly different than those of $\mathrm{CO} / \mathrm{H}_{2}$. Hawkes et al. observed monomodal PDFs of reactive scalars (e.g., $\mathrm{OH}$ ) during extinction and reignition, whereas we have observed bimodal behavior of PDFs of reactive scalars for the ethylene flame during extinction. These results are consistent with the experimental studies of Masri and Bilger, who considered non-premixed flame extinction with $\mathrm{H}_{2}$, $\mathrm{CO} / \mathrm{H}_{2}$, and $\mathrm{CH}_{4}$ flames [12]. $\mathrm{CO} / \mathrm{H}_{2}$ has a broader reaction zone width in the mixture fraction coordinate and a lower activation energy than ethylene, making $\mathrm{CO} / \mathrm{H}_{2}$ a more robust fuel in terms of its extinction and reignition characteristics. Important differences in the flame structure and resulting reignition processes may result.

In this paper, we present results of the DNS simulations to quantify the effect of the varying Damköhler number on the evolution of the jet flame and the combustion processes. These results include extent of extinction, scalar dissipation rate profiles, and flame surface area. Non-premixed and premixed modes of flame reignition are considered using a flame index. A progress variable is considered [13], and dissipation terms and reaction terms of its transport equation are investigated a-priori.

\section{Numerical implementation}

The following sections describe the DNS code implementation, along with the configuration of the cases and the chemical mechanism employed.

\subsection{DNS Code description}

The DNS code used in the simulations is called S3D [14]. The code is written in Fortran 90 and parallelized with MPI. S3D solves the compressible, reacting Navier-Stokes equations given by the following equations (in index notation) for continuity, momentum, energy, and species mass fractions, respectively:

$$
\begin{aligned}
& \frac{\partial \rho}{\partial t}=-\frac{\partial\left(\rho v_{i}\right)}{\partial x_{i}} \\
& \frac{\partial\left(\rho v_{i}\right)}{\partial t}=-\frac{\partial\left(\rho v_{j} v_{i}\right)}{\partial x_{i}}-\frac{\partial P}{\partial x_{i}}+\frac{\partial \tau_{i, j}}{\partial x_{i}},
\end{aligned}
$$

$$
\begin{aligned}
& \frac{\partial\left(\rho e_{0}\right)}{\partial t}=-\frac{\partial\left(\rho e_{o} v_{i}\right)}{\partial x_{i}}-\frac{\partial\left(P v_{i}\right)}{\partial x_{i}}+\frac{\partial\left(\tau_{i, j} \cdot v_{j}\right)}{\partial x_{i}}-\frac{\partial q_{i}}{\partial x_{i}}, \\
& \frac{\partial\left(\rho Y_{k}\right)}{\partial t}=-\frac{\partial\left(\rho Y_{k} v_{i}\right)}{\partial x_{i}}-\frac{\partial\left(j_{k, i}\right)}{\partial x_{i}}+\omega_{k} .
\end{aligned}
$$

In these equations, $\rho$ is density, $v_{i}$ is velocity, $t$ is time, $x_{i}$ is position, $P$ is pressure, $\tau_{i, j}$ is the viscous stress tensor, $Y_{k}$ is the mass fraction of species $k$, and $\omega_{k}$ is the species reaction rate. In addition, $e_{o}, q_{i}$, and $j_{k, i}$ are the specific total energy, heat flux vector, and species diffusion flux vector, respectively. The total energy is given by $e_{o}=v_{i} v_{i} / 2+h-P / \rho$, and the heat flux vector is given by $q_{i}=-\lambda \nabla T+\sum_{k} h_{k} j_{k, i}$, where $\lambda$ is the thermal conductivity, $T$ is temperature, and $h$ and $h_{k}$ are the specific enthalpies of the mixture and species, respectively. The transport equations are closed with constitutive relations for the species diffusivities, the stress tensor, a thermodynamic relation linking enthalpy and temperature, $h=h\left(T, Y_{k}\right)$, and the ideal gas equation of state $P=\rho R T / M$, where $M$ is the gas mixture molecular weight, and $R$ is the universal gas constant. The viscous stress tensor is computed as $\tau_{i, j}=\mu\left(\frac{\partial v_{i}}{\partial x_{j}}+\frac{\partial v_{j}}{\partial x_{i}}-\frac{2}{3} \delta_{i, j} \frac{\partial v_{l}}{\partial x_{l}}\right)$. A mixture-averaged formulation based on a form of Fick's law is used for the species diffusion flux $j_{k, i}=-\rho D_{k} \frac{\partial Y_{k}}{\partial x_{i}}-\frac{\rho D_{k} Y_{k}}{M} \frac{\partial M}{\partial x_{i}}$, where $D_{k}$ is a mixture-averaged diffusion coefficient computed using the Chemkin Transport package [15]. The diffusion flux of nitrogen is computed as one minus the sum of the diffusion fluxes for the other species in order to enforce a null sum of diffusion fluxes.

S3D integrates the reacting flow equations, given above, using a fourth-order, six-stage explicit Runge-Kutta method [16]. Spatial derivatives are approximated on a finite difference grid using eighth-order central differences. A 10th spatial filter is applied at each timestep to remove high wavenumber content and reduce aliasing errors [17]. Non-reflecting outflow boundary conditions are employed at open boundaries using the formulation of Sutherland and Kennedy [18].

\subsection{Combustion chemistry}

The present study considers flames with intense finite rate chemistry associated with turbulent extinction and reignition under a wide range of stoichiometric conditions, and flame modes (both non-premixed and premixed). The combustion mechanism used should be accurate under these conditions, but also minimize computational costs associated with the number of chemical species transported and the cost of computing reaction rates. These costs are associated with small chemical timescales which dictate the timestep in an explicit integration scheme and lengthscales of reactive intermediates, the resolution of which dictates the computational grid size.

The chemical mechanism used for this parametric study has been described previously [19]. The mechanism is based on a validated detailed ethylene mechanism [20], in which the directed relation graph method and sensitivity analysis are used to remove species to create a skeletal mechanism, which is further reduced by defining quasi-steady state (QSS) species using computational singular perturbation. The resulting reduced mechanism consists of the following 19 transported species: $\mathrm{H}_{2}, \mathrm{H}, \mathrm{O}$, $\mathrm{O}_{2}, \mathrm{OH}, \mathrm{H}_{2} \mathrm{O}, \mathrm{HO}_{2}, \mathrm{H}_{2} \mathrm{O}_{2}, \mathrm{CH}_{3}, \mathrm{CH}_{4}, \mathrm{CO}, \mathrm{CO}_{2}, \mathrm{CH}_{2} \mathrm{O}, \mathrm{C}_{2} \mathrm{H}_{2}, \mathrm{C}_{2} \mathrm{H}_{4}$, $\mathrm{C}_{2} \mathrm{H}_{6}, \mathrm{CH}_{2} \mathrm{CO}, \mathrm{C}_{3} \mathrm{H}_{6}$, and $\mathrm{N}_{2}$. In addition, the following 10 QSS species are included in the mechanism: $\mathrm{C}, \mathrm{CH}, \mathrm{CH}_{2}, \mathrm{CH}_{2}^{*}, \mathrm{HCO}, \mathrm{CH}_{3} \mathrm{O}$, $\mathrm{C}_{2} \mathrm{H}_{3}, \mathrm{C}_{2} \mathrm{H}_{5}, \mathrm{HCCO}, \mathrm{CH}_{2} \mathrm{CHO}$. This mechanism was validated extensively against the detailed mechanism for ignition delay time, PSR extinction residence time, laminar premixed flame speed, and species profiles in premixed laminar flames and opposed jet diffusion flames. 


\subsection{DNS configuration}

The flow configuration is a temporally-evolving, planar slot-jet consisting of a central fuel core surrounded by oxidizer. The flow is periodic in the streamwise and spanwise directions, and open in the cross-stream direction, resulting in a constant bulk pressure as the flow evolves. Figure 1 shows a schematic of the configuration. The computational costs of DNS preclude the use of very large domains, and the temporal configuration allows the study of the development of the jet within the domain (in effect maximizing the residence time of flames and fluid structures in the domain by following the mean convective fluid evolution). The jet provides one direction of mean shear (in the cross-stream direction), and two statistically homogeneous directions in the streamwise and spanwise directions, maximizing the data available for statistical analysis. This configuration has been used previously in the study of turbulent soot formation [21].

The parameters of the configuration are similar to those used in a study by Hawkes et al. [11], who simulated extinction and reignition in a $\mathrm{CO} / \mathrm{H}_{2}$ jet at three Reynolds numbers, but at a constant Damköhler number. Here, we study a representative hydrocarbon fuel, ethylene, at a fixed Reynolds number of 5120, with three varying Damköler numbers. The flow conditions are those of the medium Reynolds number case of Hawkes et al.

The velocity and composition fields are initialized as follows. The streamwise velocity field is set with $\Delta U / 2$ in the central fuel stream, and $-\Delta U / 2$ in the surrounding oxidizer streams, for a velocity difference of $\Delta U$ between the streams. A mixture fraction $\xi$ profile is set with $\xi=1$ in the fuel core, and $\xi=0$ in the surrounding oxidizer. Hyperbolic tangent profiles with transition thickness

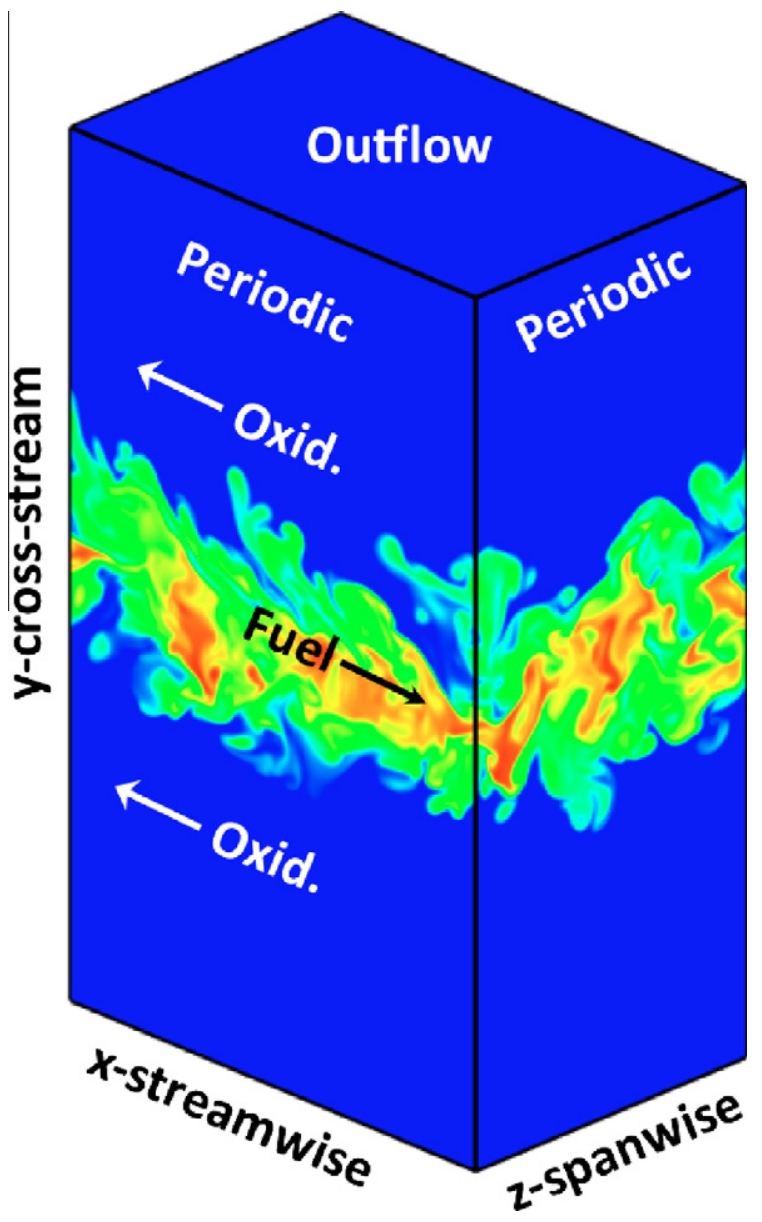

Fig. 1. Schematic of the DNS configurations.
Table 1

Simulation parameters of the three parametric DNS cases. Repeated values (e.g., on $L_{y}$ ) refer to values for DNS Cases 1-3, respectively.

\begin{tabular}{llll}
\hline$H(\mathrm{~mm})$ & 0.96 & $L_{x} / H$ & 12 \\
$\Delta U(\mathrm{~m} / \mathrm{s})$ & 196 & $L_{y} / H$ & $15,17,19$ \\
$R e_{j e t}$ & 5120 & $L_{z} / H$ & 8 \\
$H_{\xi}(\mathrm{mm})$ & 1.5 & $\Delta x(\mu \mathrm{m})$ & 17 \\
$\delta_{u}(\mathrm{~mm})$ & 0.19 & $\delta_{\xi}(\mathrm{mm})$ & 0.74 \\
$u^{\prime} / \Delta U$ (init) & $5 \%$ & $\tau_{\text {jet }}(\mathrm{ms})$ & 0.0049 \\
$H / L_{11}$ (init) & 3 & $\tau_{\text {run }} / \tau_{\text {jet }}$ & $74,87,140$ \\
\hline
\end{tabular}

$\delta$ are used as smooth transitions between the fuel and oxidizer streams, and between the positive velocity in the jet core and the negative velocity surrounding the core. Table 1 gives the geometric parameters used in the three parametric cases. $H$ and $H_{\xi}$ are the velocity jet height and the fuel core height, respectively. That is, $H_{\xi}$ is the thickness of the central fuel core, and $H$ is the thickness of the velocity jet. $\delta$ is the transition thickness of the velocity profile, and $\delta_{\xi}$ is the transition thickness of the mixture fraction profile. $L_{x}, L_{y}$, and $L_{z}$ are the domain lengths in the $x, y$, and $z$ directions, respectively. The $x, y$, and $z$ directions correspond to the streamwise, cross-stream, and spanwise directions, respectively. $\Delta x$ is the grid spacing (uniform and equal in all three directions), and $\tau_{j e t}$ is the jet timescale defined as $H / \Delta U$. A homogeneous, isotropic turbulent velocity profile is overlaid on the streamwise velocity profile in the region of the velocity core $(\mathrm{H})$ to trip the shear layers. The turbulence intensity $\left(u^{\prime}\right)$ and integral lengthscale $\left(L_{11}\right)$ parameters used in the turbulence specification are also shown in the table. The turbulence intensity and integral timescale $\left(L_{11} / u^{\prime}\right)$ are small compared to the shear velocity, and jet timescale. The shear layers develop rather quickly, and velocity fluctuations rise substantially within $7 \tau_{\text {jet }}$. Figure 2 shows the initial condition at a streamwise and cross-stream $(x-y)$ plane of the flow. Smoothed temperature contours are shown in grayscale. The $z$-velocity field from the initial turbulence is shown as contours in the jet core. Four horizontal lines are also shown. The inner two lines indicate the velocity core $H$, and the outer two lines indicate the fuel core $H_{\xi}$. Note that the temperature peaks outside the initial turbulence field at the stoichiometric point.

The gas composition is specified through the mixture fraction profile. A steady laminar flamelet solution (assuming unity Lewis numbers) is mapped to the domain with a consistent scalar dissipation rate profile between the flamelet solution and the mixture fraction profile in the DNS [19]. The profile width $\delta_{\xi}$ corresponds to $50 \%$ of the steady laminar extinction width.

It was desired to match as many flow/flame parameters as possible between the previous $\mathrm{CO} / \mathrm{H}_{2}$ simulation [11], and the present cases. The parameters of interest are the stoichiometric mixture fraction, the density ratio of the reactants and products, the

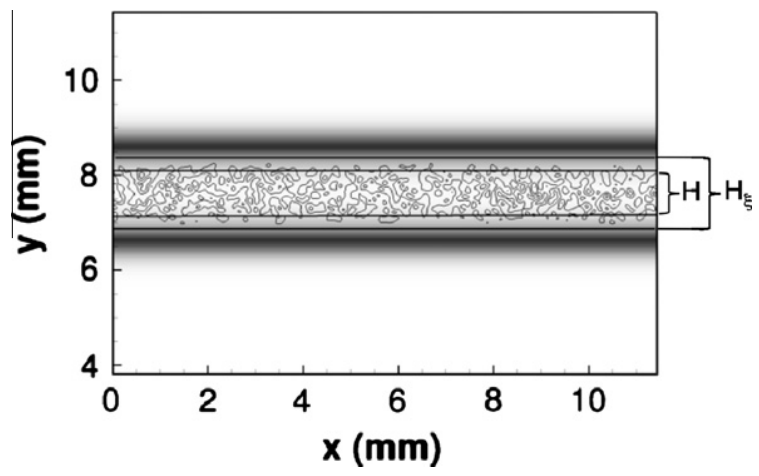

Fig. 2. Initial profile of temperature (increasing white to black), with $z$-velocity contours and horizontal lines demarcating the jet core for velocity (inner lines), and mixture fraction (outer lines). 
Table 2

Comparison of fuel parameters.

\begin{tabular}{llllll}
\hline & $\mathrm{CO} / \mathrm{H}_{2}$ & & $\mathrm{C}_{2} \mathrm{H}_{4}$ & & \\
\cline { 3 - 6 } & & & Case 1 & Case 2 & Case 3 \\
\hline$\xi_{s t}$ & 0.422 & 0.422 & 0.17 & 0.17 & 0.17 \\
$T_{o}(\mathrm{~K})$ & 500 & 550 & 550 & 550 & 550 \\
$\rho_{r} / \rho_{p}$ & 4.2 & 4.3 & 5.2 & 5.0 & 4.8 \\
$v\left(\mathrm{~cm}^{2} / \mathrm{s}\right)$ & 0.416 & 0.421 & 0.35 & 0.36 & 0.37 \\
$\chi_{q}(1 / \mathrm{s})$ & 2380 & 2380 & 4774 & 3587 & 2380 \\
$T_{q}(\mathrm{~K})$ & 1296 & 1700 & 1953 & 1896 & 1822 \\
$T_{\text {ad }}(\mathrm{K})$ & 2376 & 2345 & 2721 & 2660 & 2569 \\
\hline
\end{tabular}

kinematic viscosity, and the extinction scalar dissipation rate. Table 2 shows a comparison of these parameters between the fuels. The density ratio reported is for reactants and adiabatic equilibrium products of a stoichiometric mixture of the fuel and oxidizer streams. The kinematic viscosity is reported for the fuel stream. The scalar dissipation rate $\chi_{q}$ shown is the stoichiometric value at flame extinction for an adiabatic, steady laminar flamelet solution using the same chemical mechanism as in the DNS, but with unity Lewis numbers. The corresponding stoichiometric flame temperature at the extinction limit $T_{q}$ is also shown. The flamelet solutions were obtained solving the unsteady problem to steady state in the mixture fraction coordinate, and beginning each calculation with the solution at a lower scalar dissipation rate as the extinction dissipation rate was approached. The first two columns in the table compare $\mathrm{CO} / \mathrm{H}_{2}$ and ethylene with the same value of the stoichiometric mixture fraction. The stoichiometric extinction scalar dissipation rate for these two cases (as well as Case 3 ) is the same. This was achieved in the ethylene cases by varying the total $\mathrm{N}_{2}$ in the system. That is, the stream compositions were varied so that, at the stoichiometric point, the relative concentration of nitrogen to fuel was changed. The stoichiometric mixture fraction was varied by moving $\mathrm{N}_{2}$ from the oxidizer stream to the fuel stream [22]. Note the remarkable agreement between the parameters of these two cases. The quench temperature of the ethylene case is much higher than that of the $\mathrm{CO} / \mathrm{H}_{2}$ case.

In test simulations (run at lower grid resolution to reduce computational costs), the ethylene case at $\xi_{s t}=0.422$ resulted in global extinction, and the value of $\xi_{s t}$ had to be reduced to 0.17 . This effectively moves the flame further from the highest shear regions of the jet. Figure 3 shows the moles of $\mathrm{N}_{2}, \mathrm{O}_{2}$, and fuel $\left(\mathrm{C}_{2} \mathrm{H}_{4}\right.$, or $\mathrm{CO} /$ $\mathrm{H}_{2}$ ) at the stoichiometric point for the three cases considered here, along with the $\mathrm{CO} / \mathrm{H}_{2}$ case, and that for an ethylene air mixture. The graph is on a basis of one mole of fuel, with 0.5 and 3 moles $\mathrm{O}_{2}$ for the $\mathrm{CO} / \mathrm{H}_{2}$ and ethylene cases, respectively. The moles of $\mathrm{N}_{2}$ in the graph are $2.1667,6.870,7.927,9.516$, and 11.28 moles $\mathrm{N}_{2}$ for the $\mathrm{CO} / \mathrm{H}_{2}$ fuel, Cases $1,2,3$, and ethylene/air, respectively. The corresponding stream compositions for Cases 1-3 are given

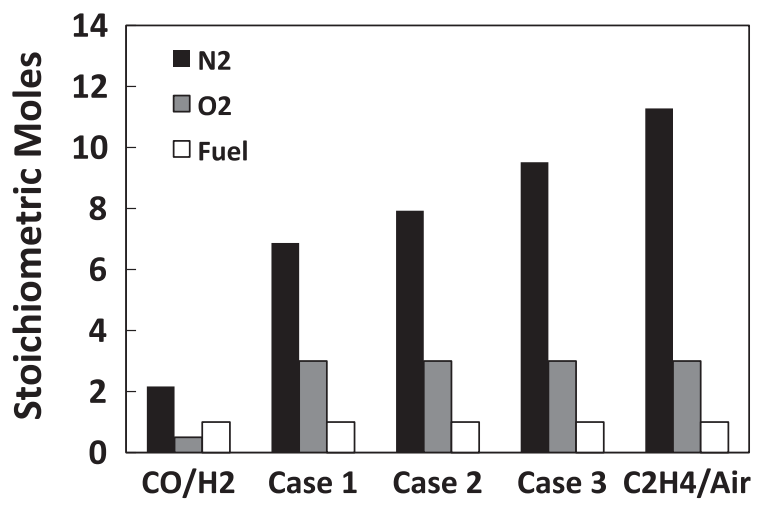

Fig. 3. Stoichiometric composition in terms of moles on a one mole fuel basis for several cases.
Table 3

Stream compositions (mole fractions) for the three cases.

\begin{tabular}{lllll}
\hline & & Case 1 & Case 2 & Case 3 \\
\hline$\xi=0$ & $\mathrm{O}_{2}$ & 0.33516 & 0.30525 & 0.26914 \\
& $\mathrm{~N}_{2}$ & 0.66484 & 0.69475 & 0.73086 \\
$\xi=1$ & $\mathrm{C}_{2} \mathrm{H}_{4}$ & 0.52105 & 0.47642 & 0.47205 \\
& $\mathrm{~N}_{2}$ & 0.47895 & 0.52358 & 0.57795 \\
\hline
\end{tabular}

in Table 3. The mole ratio of $\mathrm{CO}$ to $\mathrm{H}_{2}$ for the syngas fuel is $5: 1$ as reported in [11].

For the three simulation cases $1-3$, the density ratios, kinematic viscosities, and quench and adiabatic temperatures vary by only $3-$ $4 \%$ from their respective means. This, together with the observation that the combustion rates are dominated by turbulent mixing rates up to the point of extinction, results in a sole parameter, $\chi_{q}$, determining the degree of extinction, reignition, and flow evolution for the three cases studied.

In Table 2, the configuration parameters for the three cases studied are shown. Each case is initialized with the same $\xi_{s t}$, but the stoichiometric extinction scalar dissipation rate (steady laminar value) is varied by adjusting the nitrogen concentration of the streams as noted above. This results in Damköhler numbers $D a$ of 0.023 , 0.017 , and 0.011 for Cases $1-3$, respectively, where $D a=\chi_{q} \tau_{j e t}$. Case 1 experiences the least extinction, and Case 3 the most. This definition of Damköhler number was used by Hawkes et al. [11]. Definition of an intrinsic chemical timescale is difficult for detailed chemical mechanisms. Here, the intrinsic chemical timescale is defined as the inverse of the stoichiometric steady laminar flamelet extinction scalar dissipation rate. For steady flamelets at extinction, the mixing rate, characterized by $\chi$, is balanced by the chemical reaction rate, and increases in $\chi$ result in flame extinction. Hence, $1 / \chi_{q}$ is a reasonable estimate of the chemical timescale, especially considering the present emphasis on flame extinction.

\section{Simulation results}

In the following sections, results of the three parametric simulations are presented in terms of general behavior and observations of the flame structure, heat release rates, the degree of extinction, followed by an analysis of the flame reignition mechanisms for the three cases, and analysis of a flame progress variable.

\subsection{Extinction effects on jet evolution}

The purpose of the parametric simulations is to vary the degree of flame extinction achieved under nearly identical flow conditions (constant Re), through variation in the Damköhler number. The Damköhler number varies over a factor of two and a wide range of flame extinction is observed in the three cases with Case 1 exhibiting a modest degree of extinction, Case 2 experiencing significant extinction, and Case 3 experiencing near blowout conditions. Three regimes exist in the development of the flow:

1. Shear layer development and flame-turbulence interaction.

2. Flame extinction with mixing of fuel, oxidizer, and combustion products.

3. Reignition of the turbulent flame.

Figure 4 shows contour plots of temperature at the point of maximum extinction for the three cases $(t=0.14,0.18$, and $0.38 \mathrm{~ms}$, for Cases $1-3$, respectively). The stoichiometric isocontour of mixture fraction is overlaid. The figures show a highly contorted stoichiometric surface with regions of burning and extinguished flame zones. The degree of flame extinction will influence the degree of mixing between fuel, air and combustion products prior 

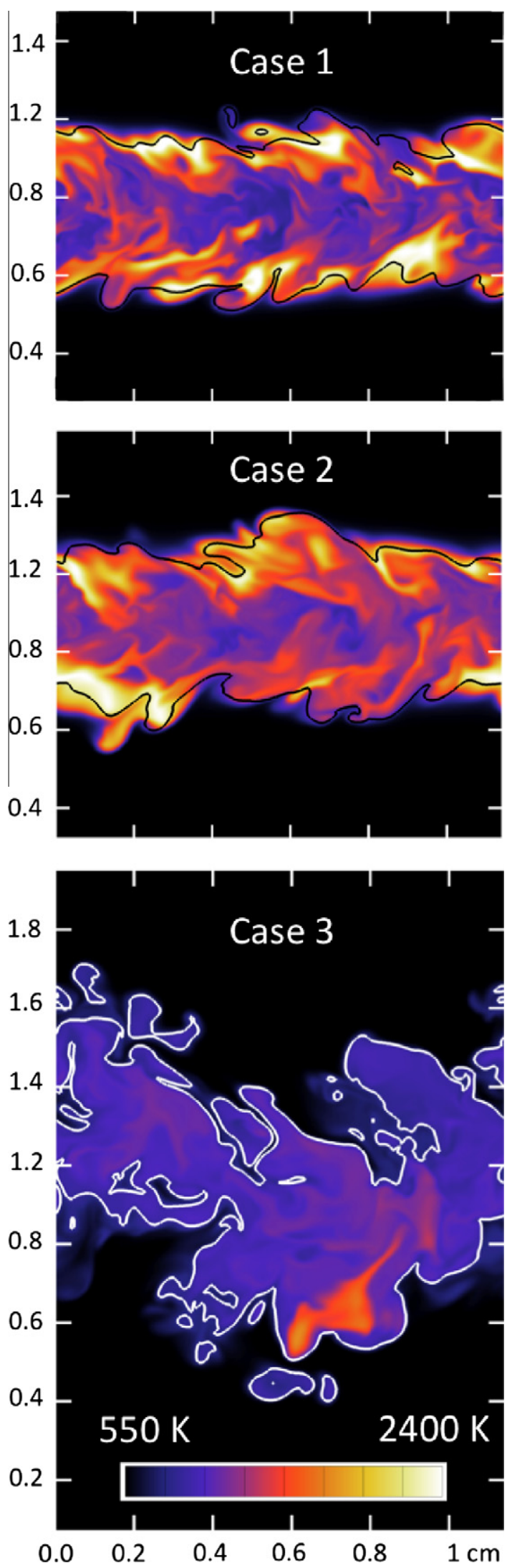

Fig. 4. Temperature contours for three cases.

to reignition, which may have a significant effect on the rate and mode of reignition. In Case 3, the extinction is nearly complete, with all but a single, hot reacting flame kernel surviving the strain- ing velocity field. As the scalar dissipation rate relaxes after flame extinction, the single kernel, shown in the lower center region of the plot for Case 3, grows to reignite the turbulent flame.

Figure 5 shows mean temperature profiles conditioned on the mixture fraction as a function of mixture fraction and time for the three cases, along with the conditional mean standard deviation of temperature. In all three cases, the temperature is high initially, then decreases to a minimum at the time of maximum extinction, then rises again as the flame strain relaxes and reignition occurs. The degree of extinction is again shown to be much higher for Case 3 as observed from these conditional mean temperature profiles. The conditional standard deviations for Cases 2 and 3 are similar. The values are low prior to the onset of extinction, then rise to a maximum around the level of maximum extinction, and subsequently decrease again as the flame reignites and the scalar dissipation rate decreases. In contrast, the conditional mean standard deviation of temperature for Case 3 begins low, rises to a maximum as the flame is extinguished, then decreases again as the flame nearly disappears and the mixture is homogenized somewhat, followed by an increase in deviation through reignition. Reignition for Case 3 is incomplete as noted by the relatively low conditional mean temperature for this case at the end of the simulation. If the simulation were carried out longer, the conditional standard deviation would likely decrease again as the flame fully reignites. Figure 5 also shows a continual decrease in the peak value of the mixture fraction as the fuel core is mixed out. By the end of the simulation, the peak mixture fraction in the domain is approximately $0.7,0.6$, and 0.3 for Cases $1-3$, respectively.

The conditional mean stoichiometric scalar dissipation rate as a function of time for the three cases is shown in Fig. 6. Each of the curves begins at the initial scalar dissipation rate. The curves then decrease slightly as the flames relax, prior to the onset of turbulence-flame interactions. As the turbulence developes, the mean scalar dissipation rate increases, reaches a maximum, then decreases as the turbulence intensity relaxes. While the curves for the three cases are qualitatively similar, they show significant quantitative differences even though the flow parameters (velocity, geometric properties) are the same for the three cases. Early on, the three curves are nearly identical. The peak values for Cases 1 and 2 are close in value, while the peak for Case 3 is significantly higher than the other cases. The dashed lines in Fig. 6 correspond to the steady laminar extinction values for the three cases. The peak mean dissipation rate for Case 1 occurs at approximately the same value as the steady laminar extinction value, while the mean of Case 2 is somewhat above the value and Case 3 is more than twice as high as the laminar extinction value. The width of the profiles in time increases for Cases $1-3$, so that Case 3 experiences a higher dissipation rate for a longer time. The increasing width of the profiles results in cases with greater extinction residing above the laminar extinction values for longer times, delaying the onset of the reignition processes. This increased scalar dissipation rate is due to the increased level of flame extinction. These results are consistent with a local increase in the Reynolds number, hence scalar dissipation rate, associated with decreased viscosity as the stoichiometric temperature decreases through flame extinction. In addition, increased flame extinction results in decreased flow dilatation and density ratios due to reduced global heat release rates, contributing to higher scalar dissipation rates. Pantano also found higher scalar dissipation rates for DNS without heat release, compared to DNS with heat release through combustion [23]. The resulting increased scalar dissipation rate with increasing extinction results in a positive feedback causing higher levels of extinction. The result is an increased sensitivity to extinction, which can lead to global flame blowout in extreme cases. The conditional root mean square of the stoichiometric scalar dissipation rate for the three cases is also shown in Fig. 6. These profiles are 
Case 1
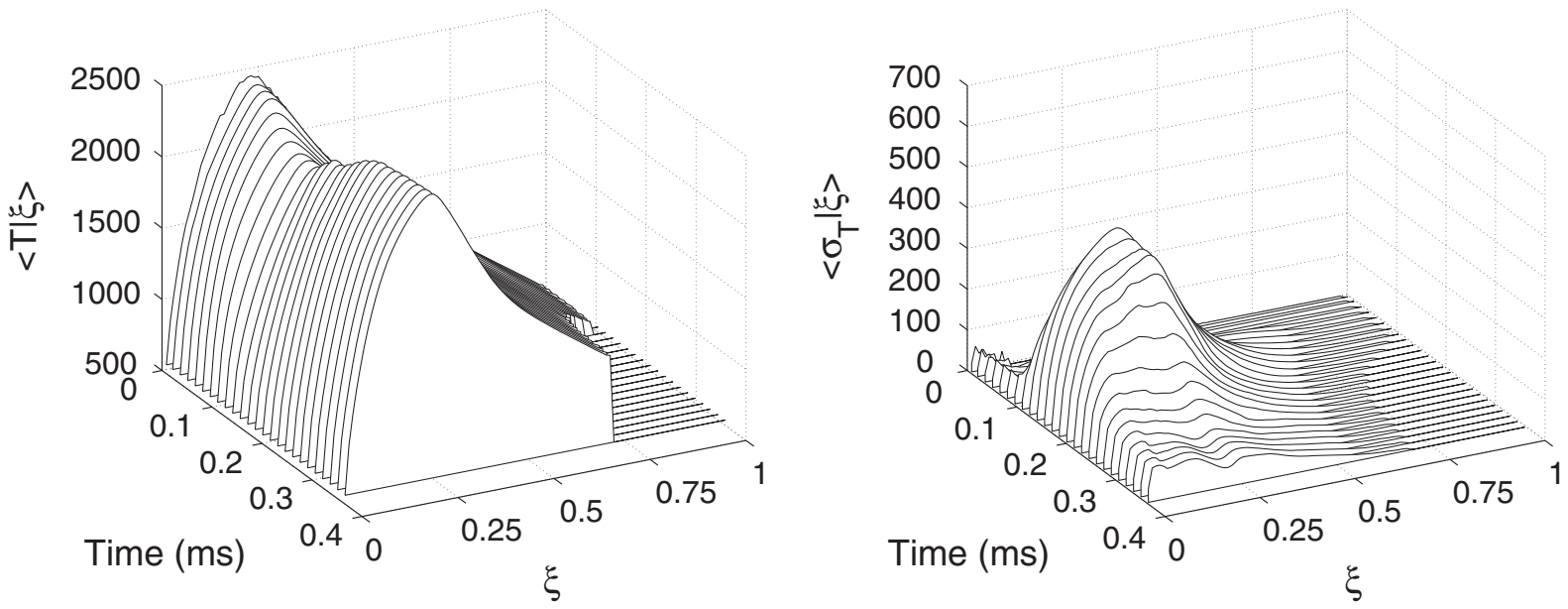

Case 2
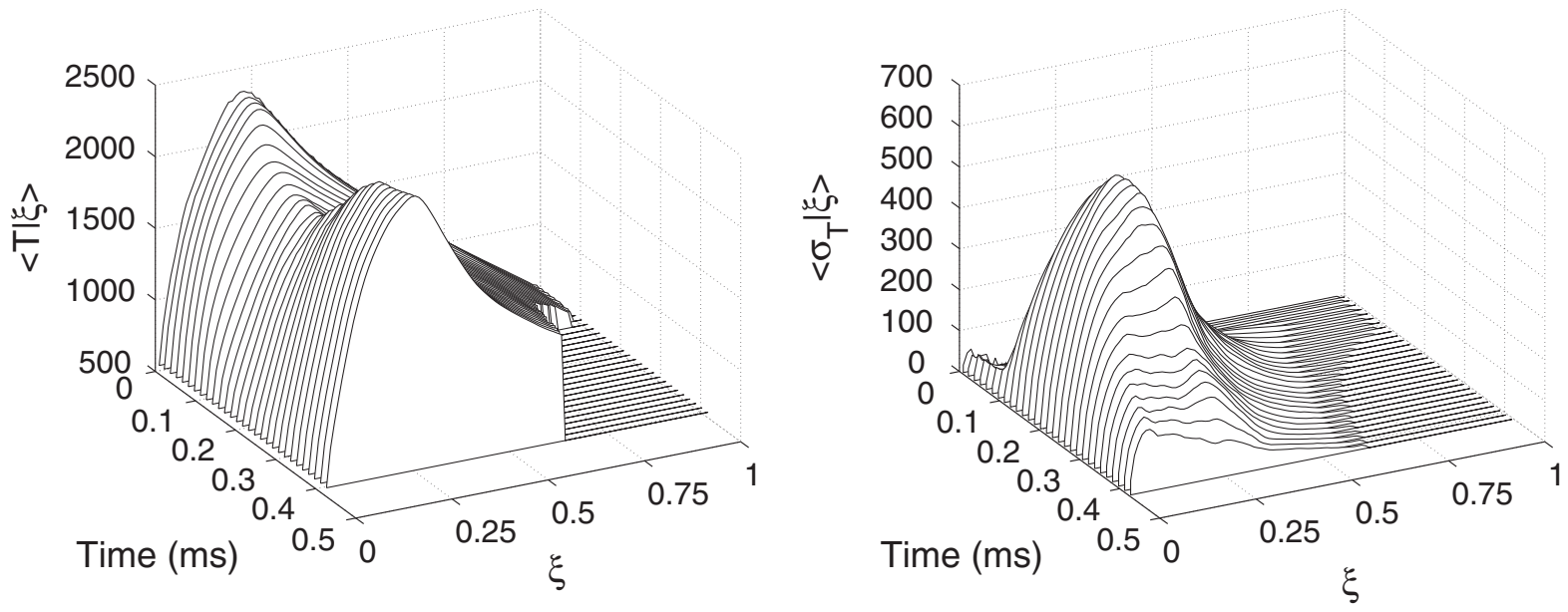

Case 3
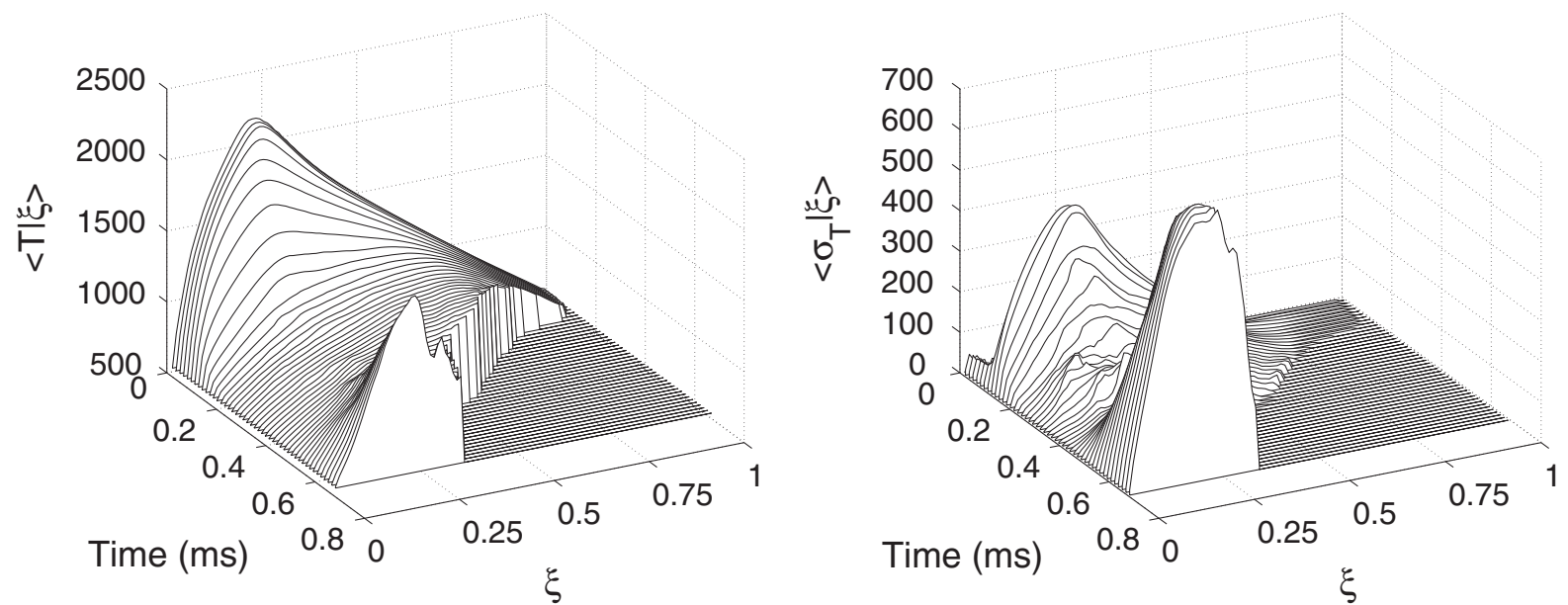

Fig. 5. Conditional mean temperature (left plots) and conditional mean standard deviation of temperature (right) plots for the three DNS cases.

very similar in both shape and magnitude to the mean values and indicate a relatively high level of conditional fluctuations in the stoichiometric scalar dissipation rate.

The PDF of the scalar dissipation rate is important in modeling of turbulent reacting flows. Figure 7 shows the PDF of $\log _{10} \chi$ conditioned on stoichiometric mixture fractions. The PDFs are pre- sented on log and linear scales for the three cases. Three times are shown for each case representing the time of peak flame extinction, the end of the simulation after flame recovery, and an intermediate time. Also shown is the normal distribution. These PDFs where constructed by extracting the stoichiometric isosurface of mixture fraction and area-weighting the resulting mixture 

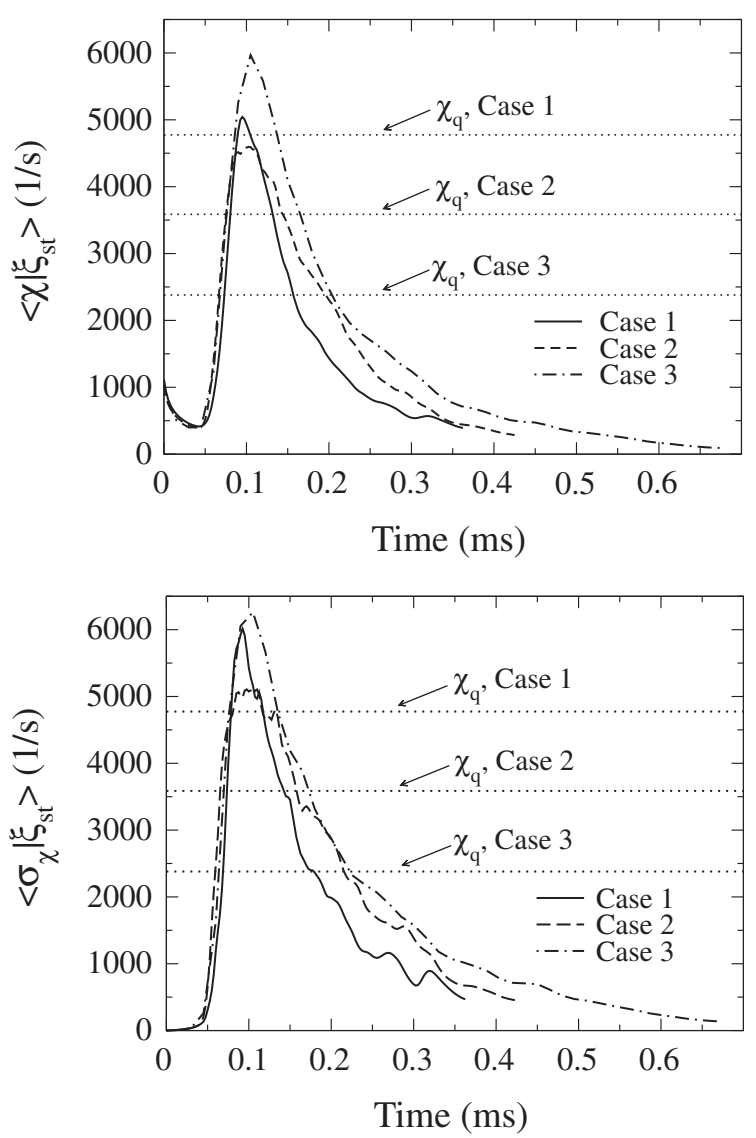

Fig. 6. Conditional means and standard deviations of stoichiometric scalar dissipation rate versus time for the three cases, along with the steady laminar extinction values (dashed lines).

fractions obtained on a triangular grid; 70 bins are used. The PDFs shown in the figure have been centered and scaled on the abscissas and scaled by the standard deviation on the ordinates. In this way, the shape of the PDFs are observed on a consistent basis. Values of the mean, variance, and skewness of $\log _{10} \chi$ are provided in Table 4 , where skewness is defined as $\left\langle(\log \chi-\langle\log \chi\rangle)^{3}\right\rangle / \sigma_{\log \chi}^{3}$. The general shape of the PDFs adhere to the lognormal distribution. However, there is some negative skewness in the profiles, which is most clearly shown on the log scale as a positive deviation from lognormal at low dissipation and a negative deviation from lognormal at high dissipation. These results are consistent with the experimental studies of Su and Clemens [24] who performed experiments of scalar mixing in inert, planar, turbulent gaseous jets. These authors concluded that the negative skewness is likely a property of the scalar dissipation rate PDF and not an artifact of experimental uncertainty or moderate Reynolds number. The DNS of Hawkes et al. [11] (which are similar to the present simulations, but with syngas as the fuel and varying Reynolds number), show the same behavior as that shown here for jet Reynolds numbers between 2510 and 9079.

The stoichiometric surface area as a function of time is shown in Fig. 8. The stoichiometric surface area is reported since reactions nominally occur on this surface, but specifically, it is reported here simply as a measure of the influence of the extent of combustion on the mixing characteristics of the flow. The surface area increases significantly as the degree of extinction increases. In Cases 1 and 2 the surface area increases as the flame extinguishes, reaches a maximum, then decreases again. In Case 1, the peak surface area occurs at $0.18 \mathrm{~ms}$, slightly later than the peak flame extinction at 0.14 ms. Case 2 is similar, but the time between the peak surface area and the peak extinction is longer: 0.3 and $0.18 \mathrm{~ms}$, respectively. These results indicate a competition between the absence of flame, which tends to increase stoichiometric area, and the presence of flame which suppresses the stoichiometric area, as the flame reignition begins on average at the point of maximum extinction. Interestingly, the peak in the stoichiometric surface area coincides with the inflection point in the reignition portion of the burning stoichiometric surface (see Fig. 9, below). In contrast, Case 3 exhibits a monotonic increase in the stoichiometric surface area over the whole simulation time, though it does appear to level off somewhat around the time of peak flame extinction $(0.38 \mathrm{~ms})$, then rises through the reignition process.

Figure 9 shows the total heat release rate of the three cases as a function of time along with the fraction of the total burning stoichiometric surface. The latter is defined as the fraction of the stoichiometric surface with an $\mathrm{OH}$ mass fraction above $50 \%$ of the steady laminar extinction value. This is a reasonable criterion as the transition from a burning to a quenched state is relatively sharp for most hydrocarbon fuels including ethylene. For Case 3, the burning fraction of the stoichiometric surface is shown as a dotted line during the reignition process after the peak extinction as the reignition does not occur as a nonpremixed flame (as discussed later). Hence, the steady laminar extinction value of the $\mathrm{OH}$ mass fraction is not consistent with the flame state. The given definition holds over the whole flame, however. The fraction of the burning stoichiometric surface gives a quantitative measure of the degree of extinction of the flame. Cases 1 and 2 experience approximately 40\%, and 70\% extinction by this measure, while Case 3 is almost completely extinguished. Additionally, Cases 1 and 2 experience nearly complete reignition of the flame, while incomplete flame reignition occurs for Case 3 by the end of its simulation.

The total heat release rate shown in Fig. 9 highlights the competition between increased heat release rate with increasing scalar dissipation rate, and decreasing heat release rate with increasing extinguished flame area. In Case 1, the total heat release rate rises to a maximum slightly later than the point of maximum extinction (the minimum in the fraction of the burning surface curve), which is slightly after the peak in the scalar dissipation rate in Fig. 6. As the dissipation rate decreases the heat release rate also decreases. In Case 2, the heat release rate initially increases as the scalar dissipation rate increases, but as the burning flame area is decreasing in this region, the reduction in the burning area competes with and dominates the increased heat release through dissipation, resulting in the small peak at a time of approximately $0.1 \mathrm{~ms}$. As the fraction of the burning surface reaches a minimum and begins to increase, the total heat release rate rises sharply at a time of $0.2 \mathrm{~ms}$. This occurs even as the dissipation rate is decreasing (Fig. 6) through a combination of the increasing flame surface area and the increasing burning fraction of that area. At a time of $0.3 \mathrm{~ms}$, a peak in the total heat release rate occurs and the value decreases as the scalar dissipation rate and the stoichiometric surface area decrease, while the fraction of the burning surface area continues to increase. Case 3 follows a similar trend to Case 2, but the burning surface area is so severely reduced that the heat release rate is low for most of the simulation before rising again through reignition at a time of approximately $0.4 \mathrm{~ms}$, nominally corresponding to the rise in the heat release rate profile of Case 2 at $0.2 \mathrm{~ms}$.

The location of the flame, extinction, and reignition processes in the mixture fraction coordinate is quantified through the heat release occurring in given regions of the mixture fraction. The heat release weighted PDF of the mixture fraction $P_{H R}(\xi)$ is shown in Fig. 10 for Cases 1-3 at six times through the evolution of each flame. The quantity $P_{H R}(\xi) d \xi$ is the fraction of the total heat release occurring in the mixture fraction range of $\xi$ to $\xi+d \xi \cdot P_{H R}(\xi)$ is defined as 

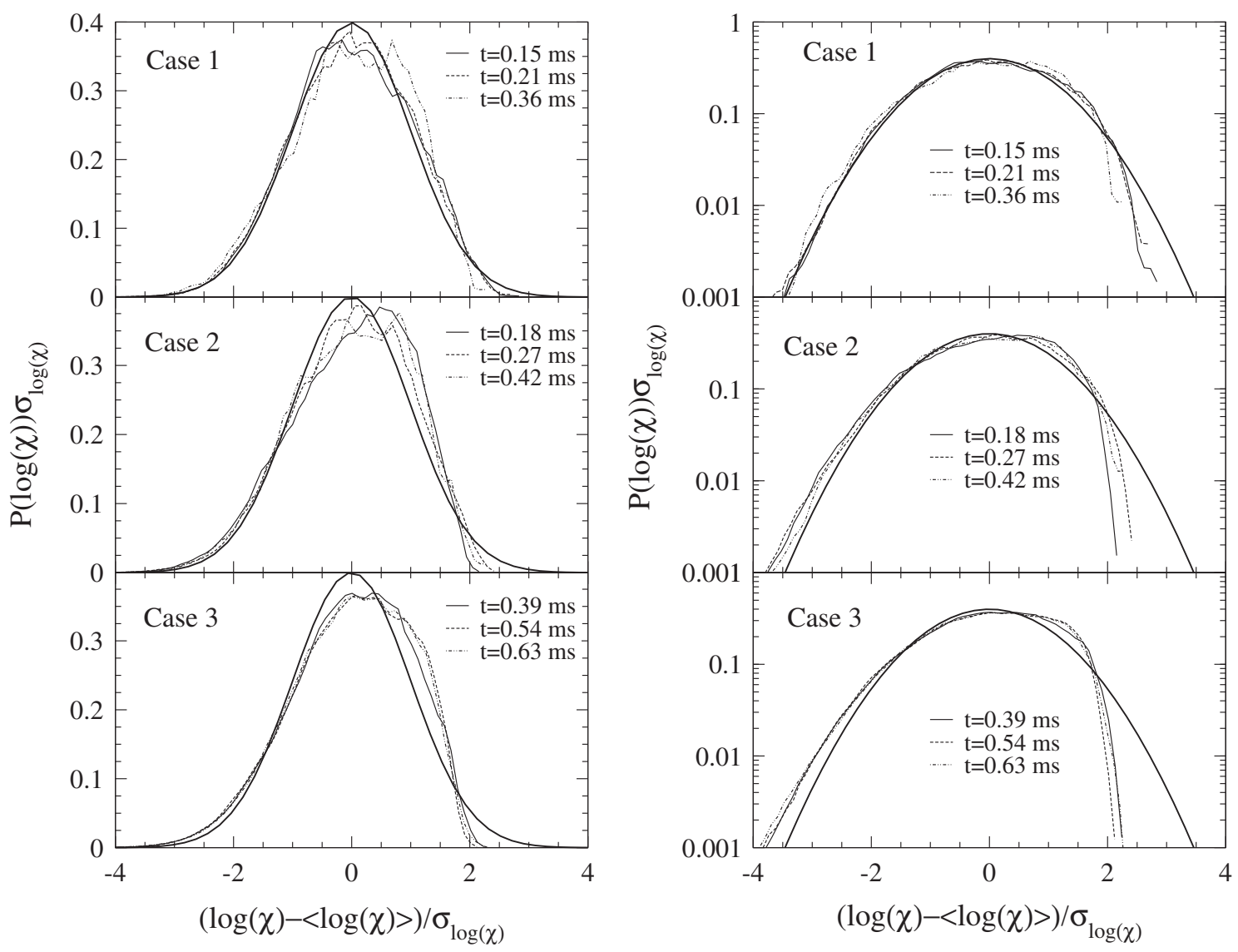

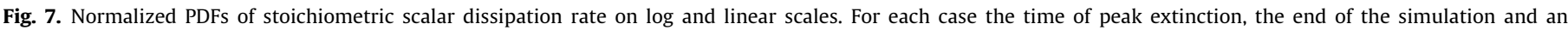
intermediate time are shown. The bold solid line is a log-normal distribution.

$P_{H R}(\xi)=\frac{\langle H R \mid \xi\rangle P(\xi)}{\langle H R\rangle}$.

Note, however, that the weighting function (heat release rate) may be positive or negative, so that the corresponding $P_{H R}(\xi)$, may also be negative, and hence calling this quantity a PDF is not strictly correct. However, if one multiplies $P_{H R}(\xi) d \xi$ by the total heat release rate, shown in Fig. 9, the result is the heat release in the range $\xi$ to $\xi+d \xi$, so that $P_{H R}(\xi)$ may be regarded as a normalized heat release density in the mixture fraction coordinate. In each of the three plots in the figure there are two graphs, and time increases for each curve from the lower graph to the upper graph, and from the dash-dotted, to the dashed, to the solid curves. The stoichiometric mixture fraction is denoted by the vertical dotted line in each plot. For each case, the peak heat release rate occurs rich of stoichiometric. The shape of the profiles in Cases 1 and 2, are similar, and relatively stationary in the mixture fraction coordinate. The peak in the heat release rate at $\xi=0.25$ is reduced at the point of maximum extinction in Cases 1 and 2 , and rises steadily through reignition. In Case 3, the heat release is initially as for Cases 1 , and 2 . After extinction in the mixing phase prior to reignition $(0.39 \mathrm{~ms})$, the heat release is positive with significant fractions of the heat release at very rich mixture fractions. The total heat release is very small during this period, however, because of the high degree of extinction. As reignition occurs, the peak heat release rate migrates progressively towards lower values of mixture fraction. Note that the peak mixture fraction in the domain at the final time is 0.3 through mixing of the fuel jet with surrounding oxidizer. This mixing effectively squeezes all reaction towards lower mixture fraction at the same time as the reignition process occurs.
Flame extinction tends to occur in regions of the stoichiometric surface with high scalar dissipation rate. In a turbulent flow in which the flame structure is strained and contorted and wrinkled the scalar dissipation rate will likely be correlated with the turbulent flow structures and the geometric properties of the stoichiometric surfaces. Figure 11 shows a top view of the stoichiometric surface (view of the jet from the oxidizer stream) in which the surface contours are $\mathrm{OH}$ mass fraction. The figures correspond to the times of peak flame extinction. Black regions are extinguished. The white contour lines shown in the figures correspond to 50\% of the steady laminar extinction value of $\mathrm{OH}$ mass fraction and delineate burning and extinguished regions. In Case 1, there is a clear visual correlation between the sign of the surface curvature and the presence of extinguished regions. The flames tend to extin-

Table 4

Mean, variance, and skewness of $\log _{10}(\chi)$ for three cases at four times.

\begin{tabular}{lllll}
\hline & Time $(\mathrm{ms})$ & $\langle\log \chi\rangle$ & $\sigma_{\log \chi}^{2}$ & Skewness \\
\hline Case 1 & 0.09 & 3.4 & 0.31 & -0.22 \\
& 0.15 & 3.2 & 0.27 & -0.14 \\
& 0.21 & 3.8 & 0.34 & -0.22 \\
Case 2 & 0.36 & 2.3 & 0.32 & -0.37 \\
& 0.09 & 3.4 & 0.25 & -0.33 \\
& 0.18 & 3.1 & 0.38 & -0.52 \\
Case 3 & 0.27 & 2.6 & 0.43 & -0.36 \\
& 0.42 & 2.0 & 0.52 & -0.38 \\
& 0.09 & 3.4 & 0.29 & -0.37 \\
& 0.39 & 2.4 & 0.46 & -0.47 \\
& 0.54 & 2.0 & 0.50 & -0.51 \\
& 0.63 & 1.7 & 0.47 & -0.49 \\
\hline
\end{tabular}




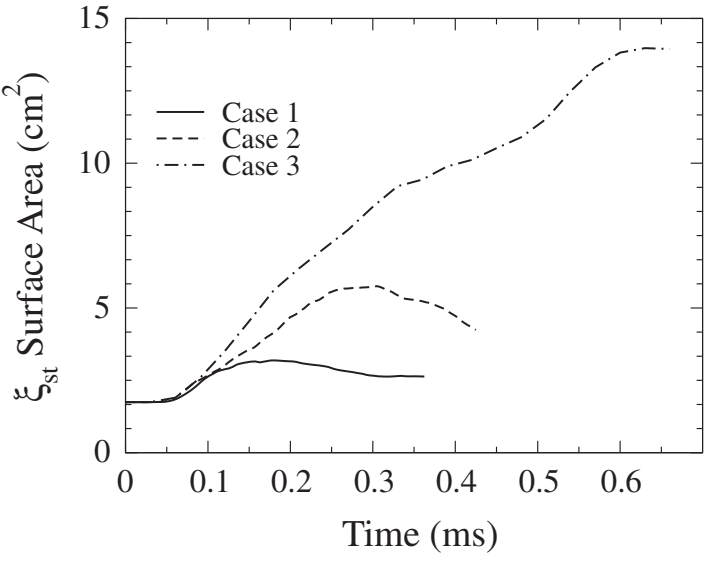

Fig. 8. Stoichiometric surface area as a function of time for the three cases.

guish first in regions where the center of curvature is in the fuel stream. The surface mean curvature is defined as $\kappa=\nabla \cdot \vec{n}$, where $\vec{n}$ is the surface normal vector directed with the defining scalar gradient. The scalar dissipation rate tends to be higher in these regions, and this correlation between scalar dissipation rate and curvature has been previously reported [21]. This is due primarily to the stoichiometric mixture fraction below 0.5 having higher dissipation rates when the center of curvature is on the fuel side than on the oxidizer side. Case 2 experiences a higher degree of extinction than Case 1, and the correlation between the orientation of curvature and extinction is less obvious at the point of maximum
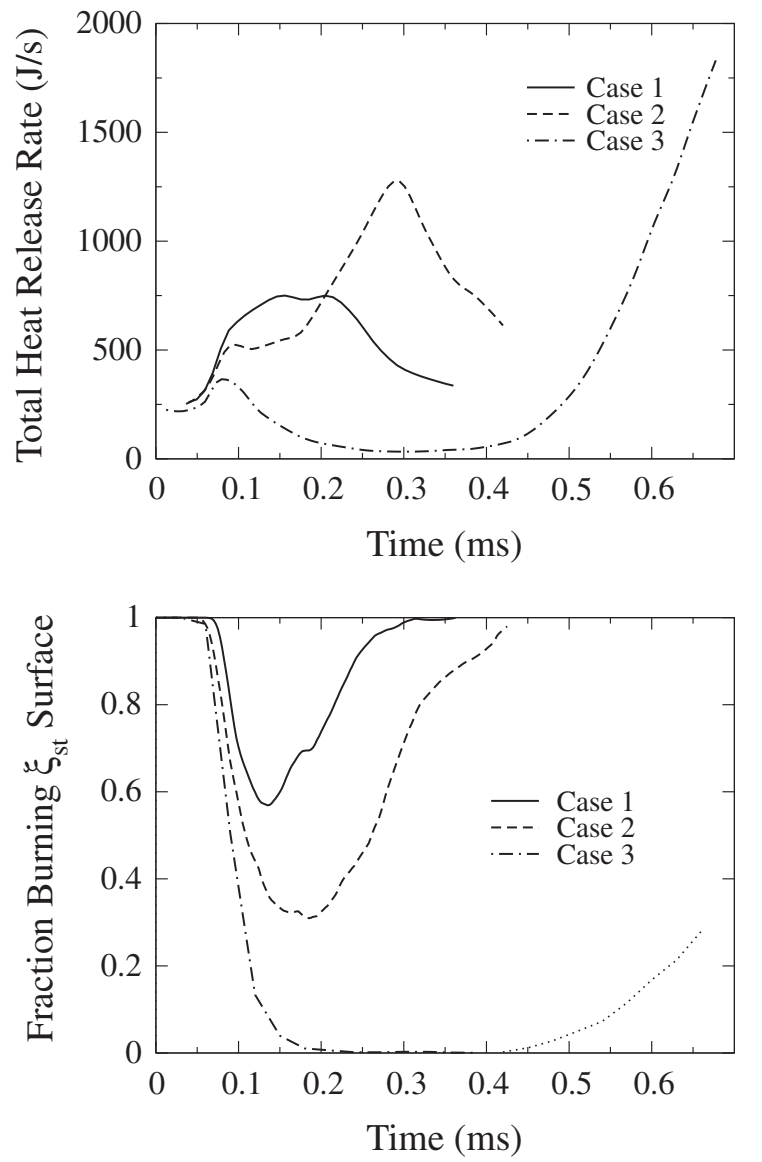

Fig. 9. Total heat release rate (top) and fraction of the total burning stoichiometric surface (bottom) versus time for the three cases.
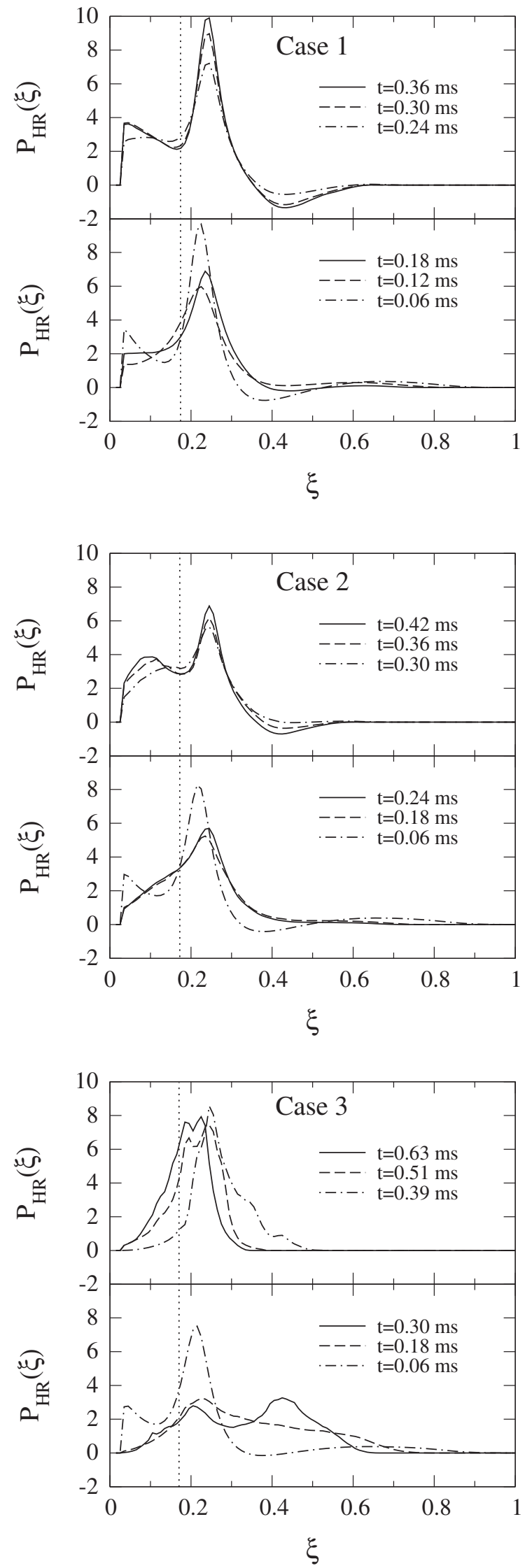

Fig. 10. Heat release weighted PDFs of mixture fraction for the three cases. Time increases from the lower to the upper graphs, from dash-dot, to dashed, to solid curves in each graph. The vertical dotted line denotes the stoichiometric mixture fraction. 

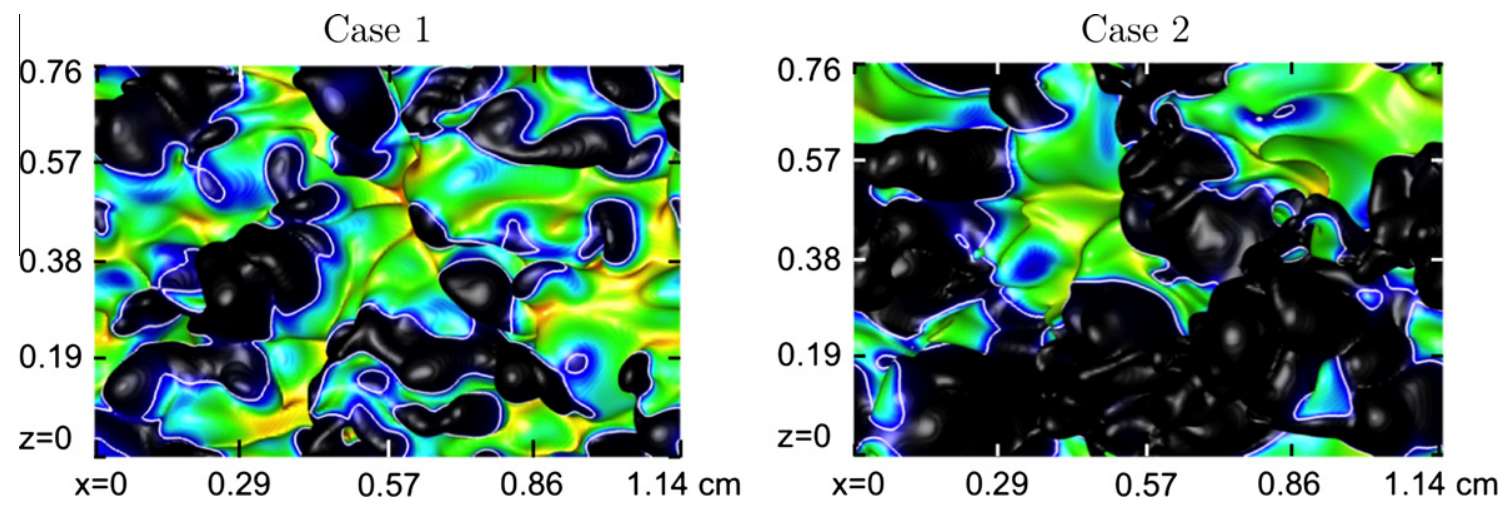

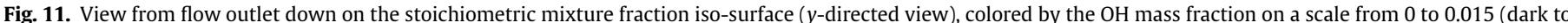

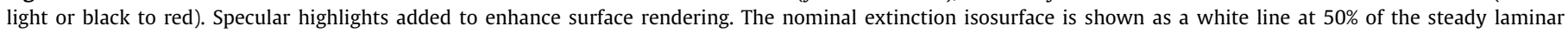
extinction $\mathrm{OH}$ mass fraction. (For interpretation of the references to color in this figure legend, the reader is referred to the web version of this article.)

extinction. Initially, the extinction occurs in regions of curvature centered in the fuel stream, but as the dissipation rate increases in time, more and more of the flame extinguishes and flame holes spread over larger areas, with less correlation of extinction with curvature.

As the stoichiometric scalar dissipation rate decreases with time (after its peak), the flame holes heal as the flame reignites. In Case 2, the rate of this healing is decreased because of the larger regions of extinction and correspondingly smaller quenched/burning interface relative to the degree of extinction. The larger regions of flame extinction in Case 2, compared to Case 1, will result in more mixing of fuel, oxidizer, and combustion products prior to reignition. This mixing complicates the description and modeling of the flame, as well as the mode of reignition. If sufficient mixing occurs prior to reignition, the reignition process may occur through a premixed flame propagation in a heterogeneous mixture. Indeed, the extreme extinction that occurs in Case 3, results in the reignition of Case 3 as a predominantly premixed flame.

\subsection{Reignition mode}

The mode in which the flame reignites can be investigated by considering the degree of alignment of fuel and oxidizer gradients through a flame index [25] defined as

$$
G_{F O}=\frac{\nabla Y_{F} \cdot \nabla Y_{O 2}}{\left|\nabla Y_{F}\right|\left|Y_{O 2}\right|} \text {. }
$$

The flame index ranges from -1 to 1 , with negative values corresponding to opposing gradients of fuel and oxidizer, as occur in non-premixed flames; positive values correspond to aligned gradients of fuel and oxidizer, as occur in premixed flames. In the calculation of the flame index, the fuel is taken as all $\mathrm{C}_{x} \mathrm{H}_{y}$ species. Figure 12 shows the cumulative heat release weighted PDF of the flame index over three ranges of the flame index. The heat release weighted PDF of flame index $P_{\gamma}\left(G_{F O}\right)$ multiplied by $d G_{F O}$ is the fraction of the heat release in the system between a flame index of $G_{F O}$ and $G_{F O}+d G_{F O}$. The cumulative PDF is taken as $\int_{G 1}^{G 2} P_{\gamma}\left(G_{F O}\right) d G_{F O}$, where $G 1$ and $G 2$ are the bounds of the integration. The three regions shown in the figure correspond to integration over angles between gradients of fuel and oxidizer aligned within $45^{\circ}$, opposed within $45^{\circ}$, and alignments intermediate between these regions. These three regions are termed R1-R3, respectively.

Initially, all three cases show perfectly opposed gradients of fuel and oxidizer consistent with the initial conditions and the nonpremixed nature of the flames. As extinction occurs, the degree of alignment increases, resulting in more of the heat release occurring in regions $\mathrm{R} 3$ and $\mathrm{R} 1$, while flames in region $\mathrm{R} 2$ decrease. As reignition of the flames occurs, Cases 1 and 2 reverse their trends, and the reaction in region $R 2$ increases, while that in regions $R 1$ and R3 decreases. Cases 1 and 2 are essentially non-premixed flames whose fuel/oxidizer gradients become more aligned through the highly turbulent processes during extinction, with greater opposition of the gradients in the initial flow and during the reignition processes. The curves for Case 1 show less deviation from opposed flow than Case 2, as this case experiences less extinction. In contrast, Case 3 begins as a non-premixed flame with all of its heat release occurring in region R2 (as for Cases 1 and 2), but as extinction and reignition occur, there is a steady shift from region $\mathrm{R} 2$ to region R1. Evidently, the reignition process occurring in Case 3 proceeds through a premixed flame mode and results in burning premixed flames. The heat release in the intermediate region R3 increases during extinction, then decreases during reignition for Case 1 . Cases 2 and 3 are similar to Case 1 in region $\mathrm{R} 1$, but the decrease in the curves occurs prior to the onset of the mean reignition process.

We note that this analysis presents an integrated picture of the reignition process in terms of the fraction of the flames existing as premixed or non-premixed flames during the flame evolution using the flame index. The specific process of reignition is complicated in that non-premixed flame reignition through edge flames cannot be distinguished locally from premixed flames due to the premixed nature of the flame tip in edge flames [26], unless a local flame structure analysis is performed. For example, Hawkes et al. [5] examined local alignments of $\mathrm{OH}$ (a flame indicator) and mixture fraction gradients. Aligned gradients indicate flame folding or premixed propagation, whereas non-aligned gradients are indicative of edge-flame propagation. The rise in the fraction of the flame index in regions R1 and R3 in Fig. 12 may be due in part to the presence of the flame holes (illustrated in Fig. 11) and corresponding edge flames. The rise in the curves in these regions is heightened by the relative increase in aligned and intermediate regions as the non-premixed opposed regions are extinguished. As the flames reignite, however, we observe the clear trend that Cases 1 and 2 recover to burning non-premixed flames, while Case 3 recovers to a burning premixed flame. This is indicated by the flame index in Fig. 12, and also by Fig. 9 where the total heat release has recover to a high value in all three cases.

The determination of the specific mechanisms of flame reignition and their statistics will require investigation into the topology of the reignition zones of individual flames. That investigation is planned for a future study, especially relating to Cases 1 and 2 . For Case 3, as noted above, the reignition proceeded from a single reaction kernel. That kernel was tracked and analysed through ignition delay computations, and surface displacement analysis 

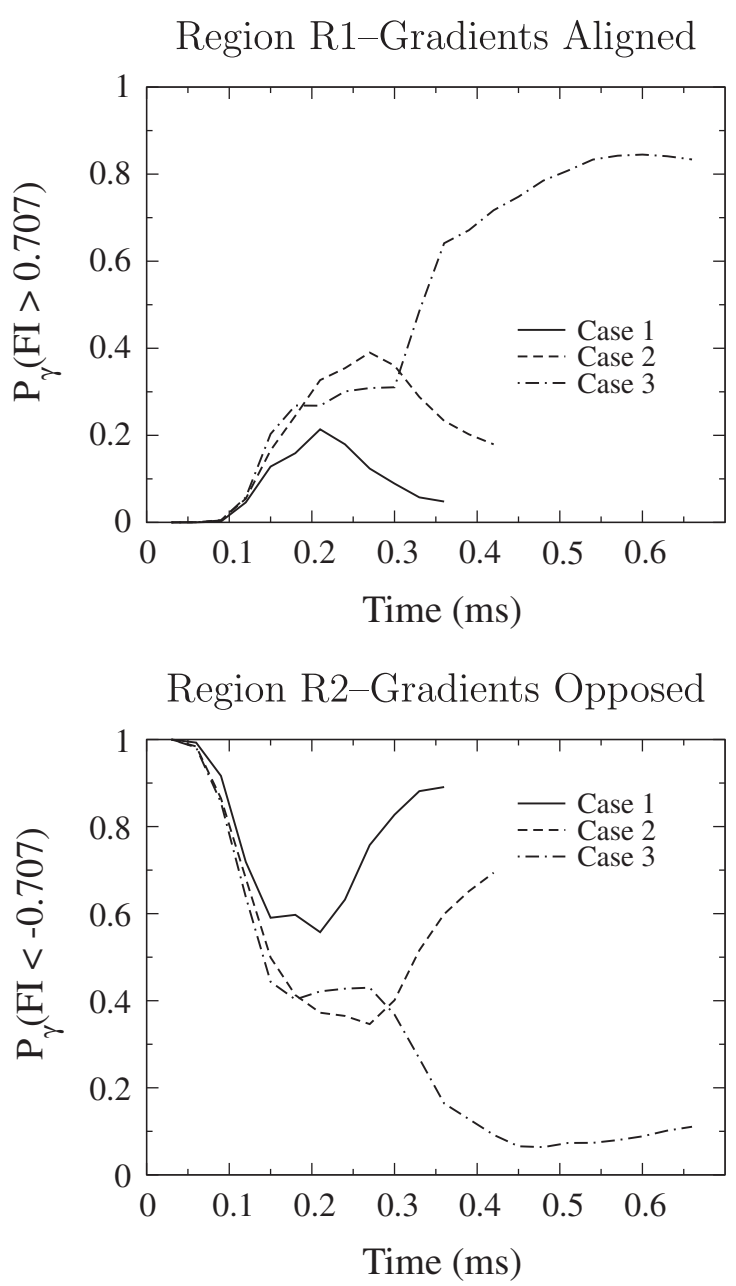

Region R3-Gradients Intermediate

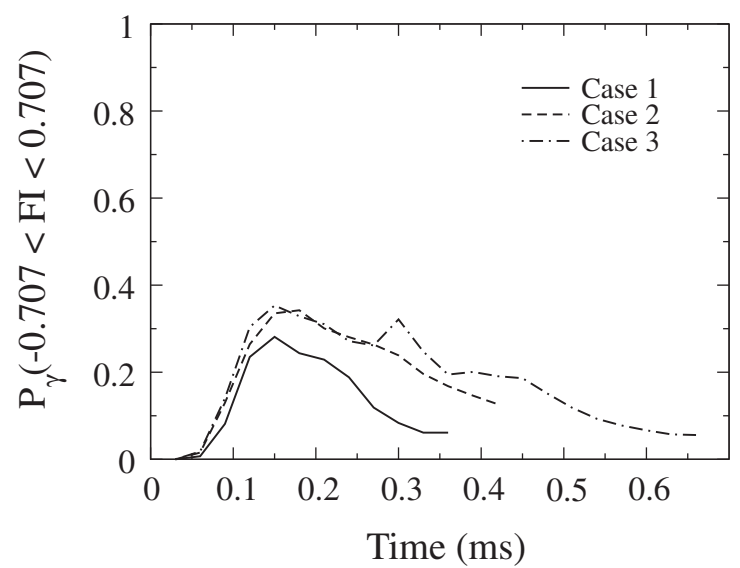

Fig. 12. Heat release weighted PDF of flame index integrated over three regions of the flame index coordinate. Region 1 corresponds to gradients of fuel and oxidizer aligned within $45^{\circ}$, Region 2 corresponds to gradients opposed within $45^{\circ}$. Region 3 is in between.

discussed below, and apparently reignites through premixed flame propagation through a stratified mixture. However, that does not preclude the presence of edge flame structures during the extinction processes prior to reignition.

A surface displacement speed analysis was performed to confirm the reignition of Case 3 as a premixed flame. The transport equation of a simple reacting scalar $\phi$ is given by
$\frac{D \phi}{D t}=-\frac{1}{\rho} \nabla \cdot \mathbf{j}_{\phi}+\frac{\omega_{\phi}}{\rho}$.

The surface velocity of an isosurface of $\phi$, say $\phi_{c}$ is given by

$s_{d}=\left.\frac{D \phi / D t}{|\nabla \phi|}\right|_{\phi=\phi_{c}}$,

where the sign convention is defined such that $s_{d}$ is positive when the isosurface is moving towards values of $\phi$ less than $\phi_{c}$. During the period of intense reignition, a value of $Y_{\mathrm{CO} 2}=0.035$ was found to closely track the peak heat release rate in the domain and this value is used as $\phi_{c}$. This value does not track the peak heat release for this case during the initial period when the non-premixed flame is extinguishing, and a general scalar that can capture both regions was not identified. However, our primary focus here is on the reignition process. The surface velocity, $s_{d}$, as defined, was computed and then normalized as $s_{d}^{*}=\frac{\rho}{\rho_{u}} s_{d}$, where $\rho_{u}$ is local unburnt density computed from the local mixture fraction and enthalpy. This definition permits direct comparison of the local isosurface velocity to the corresponding local one-dimensional, steady laminar flame speed $s_{L}$. Note that the definition of $s_{d}$ is general, and applies to both premixed and non-premixed flames. Comparison of the normalized value to the steady, laminar one-dimensional flame speed gives a direct measure of the degree to which the reignition mode occurs as a premixed flame. One-dimensional laminar flame speeds were computed using the Chemkin Premix code [27] and tabulated as a function of mixture fraction and upstream temperature. The DNS mixture fraction and enthalpy permit calculation of the corresponding unburnt (upstream) temperature and the local $s_{L}$ is interpolated from the table.

Figure 13 shows the area-weighted mean surface displacement speed $s_{d}^{*}$ as a function of time during the period of extinguished mixing and the reignition process. The corresponding average $s_{L}$ is also shown in the figure. Initially, the curve for $s_{d}^{*}$ is negative as there is little reaction at the $\phi_{c}$ surface and the $\mathrm{CO}_{2}$ field experiences strong scalar mixing. The displacement speed increases to positive values at the beginning of the reignition process after peak extinction at approximately $0.4 \mathrm{~ms}$. At times later than $0.5 \mathrm{~ms}$, significant reignition and flame propagation are occurring and the mean $s_{d}^{*}$ and $s_{L}$ are very close, indicating the premixed nature of the flames. Discrepencies between the curves in this region arise from unsteady flame propagation, multi-dimensional transport effects, or non-burning regions on the $\phi_{c}$ surface. At times prior

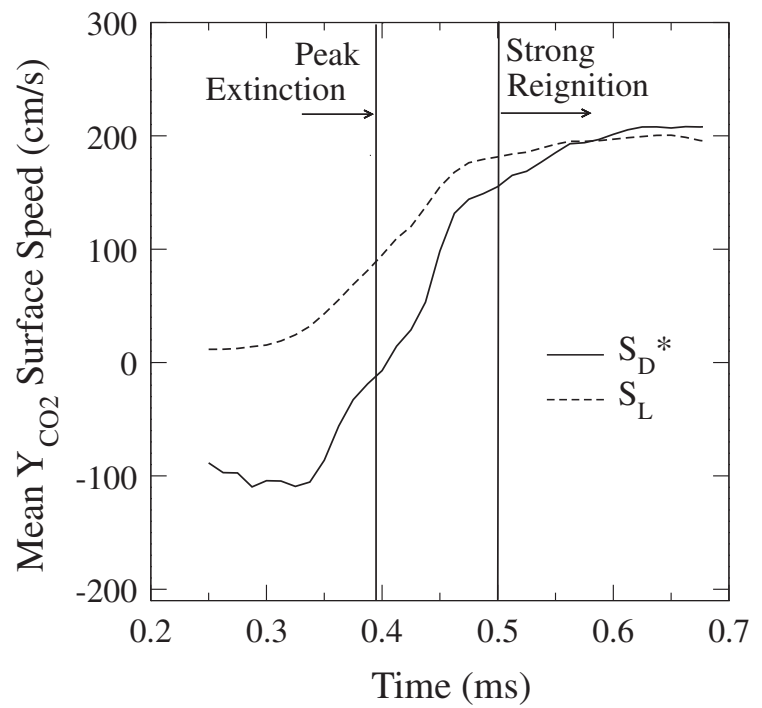

Fig. 13. Mean normalized surface velocity of $Y_{\mathrm{CO} 2}=0.035$ and corresponding laminar flame speed. 
to reignition, much of the $\mathrm{CO}_{2}$ surface is not associated with reaction, being a product of the non-premixed flames undergoing scalar mixing. Late in the simulation, nearly all of the $\mathrm{Y}_{\mathrm{CO} 2}=0.035$ coincides with the peak heat release rate. The mean $s_{L}$ curve is initially very small as the $\mathrm{Y}_{\mathrm{CO} 2}=0.035$ surface prior to extinction resides at mixture fractions of 0.04 and 0.72 , which are very lean and very rich compared to stoichiometric $(0.17)$, so that the corresponding laminar flame speed is small.

\subsection{Progress variable}

The DNS presented exhibit a high degree of extinction allowing for the possibility of partial premixing prior to reignition. Partial premixing can be important in combustion processes including lifted flames, liquid fuel sprays, and combustion in engines and gas turbines. Stratified mixtures consisting of reactants mixed with products are used as a $\mathrm{NO}_{x}$ control strategy (exhaust gas recirculation). A progress variable approach to modeling has been applied to represent partially premixed combustion $[13,28,29]$. This is done by specifying a reaction progress variable and its associated transport equation. This equation has terms that are often neglected, or require closure in LES modeling. Here, a progress variable is defined and reported for the DNS simulations, along with key terms in its transport equation.

There are many ways to define the progress variable. Typically, a given species, either a reactant or product species, or combination of species is chosen. The definition of the progress variable used here is given by

$c=\frac{Y_{i}-Y_{i}^{m i x}}{Y_{i}^{*}-Y_{i}^{m i x}}$

where $Y_{i}$ is some linear combination of species mass fractions, $Y_{i}^{*}$ is a reference state (ideally the equilibrium state of $Y_{i}$ ), and $Y_{i}^{\text {mix }}$ is $Y_{i}$ for pure mixing defined as

$Y_{i}^{m i x}=Y_{i, \xi=1} \cdot(\xi)+Y_{i, \xi=0} \cdot(1-\xi)$.

Here, we define $Y_{i}=Y_{\mathrm{CO}}+Y_{\mathrm{CO} 2}$. This combination is chosen because $\mathrm{CO}$ is the dominant product species for very rich compositions, $\mathrm{CO}_{2}$ is the dominant product species for lean compositions, and both species are present in intermediate regions. These three regions are defined as Region 1: $0 \leqslant \xi<\xi_{s t}$; Region 2: $\xi_{s t} \leqslant \xi<\xi_{s t}^{*}$; and Region 3: $\xi_{s t}^{*}<\xi \leqslant 1$. Here, $\xi_{s t}$ is the stoichiometric mixture fraction for products of complete combustion, $\xi=0.17$, and $\xi_{s t}^{*}$ is the stoichiometric mixture fraction for CO products: $\mathrm{C}_{2} \mathrm{H}_{4}+\mathrm{O}_{2} \rightarrow 2 \mathrm{CO}+2 \mathrm{H}_{2}$, with $\xi_{s t}^{*}=0.3805$. The lower curves of Fig. 14 show $Y_{i}$ at equilibrium for the three cases. Also shown are $Y_{i}$ under conditions of complete combustion products in Region 1 ; $\mathrm{CO}$ combustion products in Region 3; and linear profiles for $\mathrm{CO}$ and $\mathrm{CO}_{2}$ in Region 2, where $\mathrm{CO}$ is zero at $\xi_{s t}$ and $\mathrm{CO}_{2}$ is zero at $\xi_{s t}^{*}$. Under these conditions (used as a reference state), $Y_{i}$ is denoted $Y_{i}^{*}$, and the equilibrium state is denoted $Y_{i}^{\text {eq }}$. Note how closely $Y_{i}^{*}$ approximates $Y_{i}^{\text {eq }}$. The progress variable is defined using $Y_{i}^{*}$ since this gives piecewise-linear profiles of $Y_{i}(\xi)$, and is close to the equilibrium profile. For reference, the equilibrium progress variable, that is, the progress variable when $Y_{i}=Y_{i}^{e q}$ in Eq. (9), is also shown in Fig. 14 in the upper three curves for the three cases, with the progress variable increasing with case number.

A transport equation for the progress variable has been derived by Bray et al. [13] using the following transport equations for the mixture fraction (assuming unity Lewis numbers) and $Y_{i}$ :

$$
\begin{aligned}
& \frac{\partial \rho \xi}{\partial t}+\nabla \cdot(\rho \vec{v} \xi)=\nabla \cdot(\rho D \nabla \xi) \\
& \frac{\partial \rho Y_{i}}{\partial t}+\nabla \cdot\left(\rho \vec{v} Y_{i}\right)=\nabla \cdot\left(\rho D \nabla Y_{i}\right)+\dot{\omega}_{i}
\end{aligned}
$$

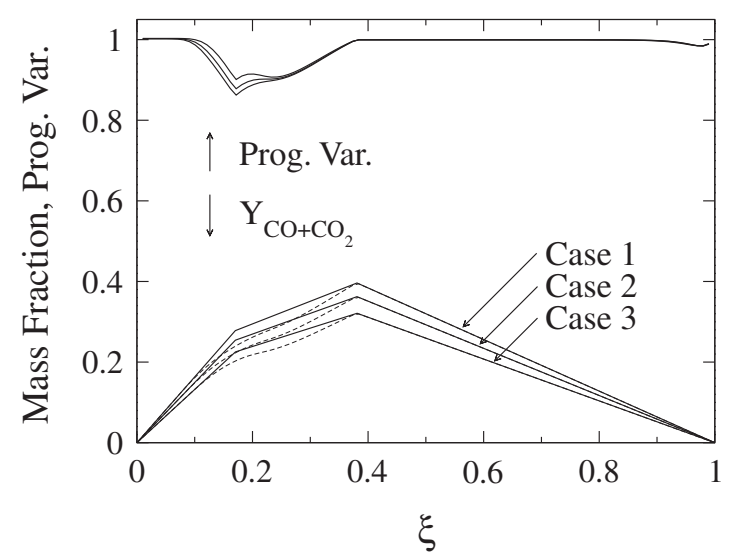

Fig. 14. $\mathrm{CO}+\mathrm{CO}_{2}$ mass fraction (lower curves) for equilibrium products (dashed lines) and products of complete combustion and combustion to CO products (solid lines). The upper plots are the equilibrium progress variable with increasing progress variable for increasing case number.

Using $Y_{i}=Y_{i}(c, \xi)$, with $c$ defined in Eq. (9), the transport equation for $c$ is derived:

$$
\begin{aligned}
& \frac{\partial \rho c}{\partial t}+\nabla \cdot(\rho \vec{v} c) \\
& \quad=\nabla \cdot(\rho D \nabla c)+\frac{1}{\partial Y_{i} / \partial c}\left[\dot{\omega}_{i}+\frac{\partial^{2} Y_{i}}{\partial c^{2}} \rho \chi_{c}+\frac{\partial^{2} Y_{i}}{\partial \xi^{2}} \rho \chi_{\xi}+\frac{\partial^{2} Y_{i}}{\partial c \partial \xi} \rho \chi_{\xi, c}\right]
\end{aligned}
$$

Here,

$$
\begin{aligned}
& \chi_{c}=D \nabla c \cdot \nabla c, \\
& \chi_{\xi}=D \nabla \xi \cdot \nabla \xi, \\
& \chi_{\xi, c}=D \nabla \xi \cdot \nabla c .
\end{aligned}
$$

In Eq. (13), the second and third terms in brackets are zero because $Y_{i}$ is linear in both $c$ and $\xi$, as defined in Eq. (9). The third term in brackets is infinite at the stoichiometric points where the piecewise-linear profiles have discontinuous slopes. Bray et al. [13] (using and equilibrium reference state) indicate that this term is approximately zero except at the stoichiometric point. This motivates the definition of $c$ in terms of $Y_{i}^{*}$, as we have done here, which is piecewise linear with mixture fraction. The remaining terms in brackets are the reaction source term and the cross dissipation term. The cross dissipation term is a measure of partial premixing and accounts for mixture fraction variations across a reaction front [29] in the sense of a propagating premixed flame, or variations of progress variable along gradients of mixture fraction in a strained diffusion flame. While definition in terms of $Y_{i}^{e q}$ may seem more physical since that is the end state of reaction, using $Y_{i}^{*}$ is reasonable as a scaling factor, and is close to $Y_{i}^{e q}$. One implication of this definition is that $c$ will not vary from zero to unity as reaction progresses from unburnt to equilibrium products, but rather from zero to the value of $c$ shown in Fig. 14, at a given mixture fraction. In practice, the reactions are far from equilibrium anyway, as shown below. The present definition of $c$ in terms of $Y_{i}^{*}$ is advantageous as there are fewer terms in the $c$ transport equation, and numerical differentiation (first and second derivatives) of the equilibrium mass fraction with respect to mixture fraction is avoided. Figure 15 shows the progress variable for steady laminar flamelet solutions for the stream compositions of Case 2 for two values of the stoichiometric scalar dissipation rate. Also shown in the figure are the progress variables for the DNS at the corresponding scalar dissipation rate, along with the DNS conditional mean profiles and steady laminar flamelet profiles of $\mathrm{CO}+\mathrm{CO}_{2}$ 

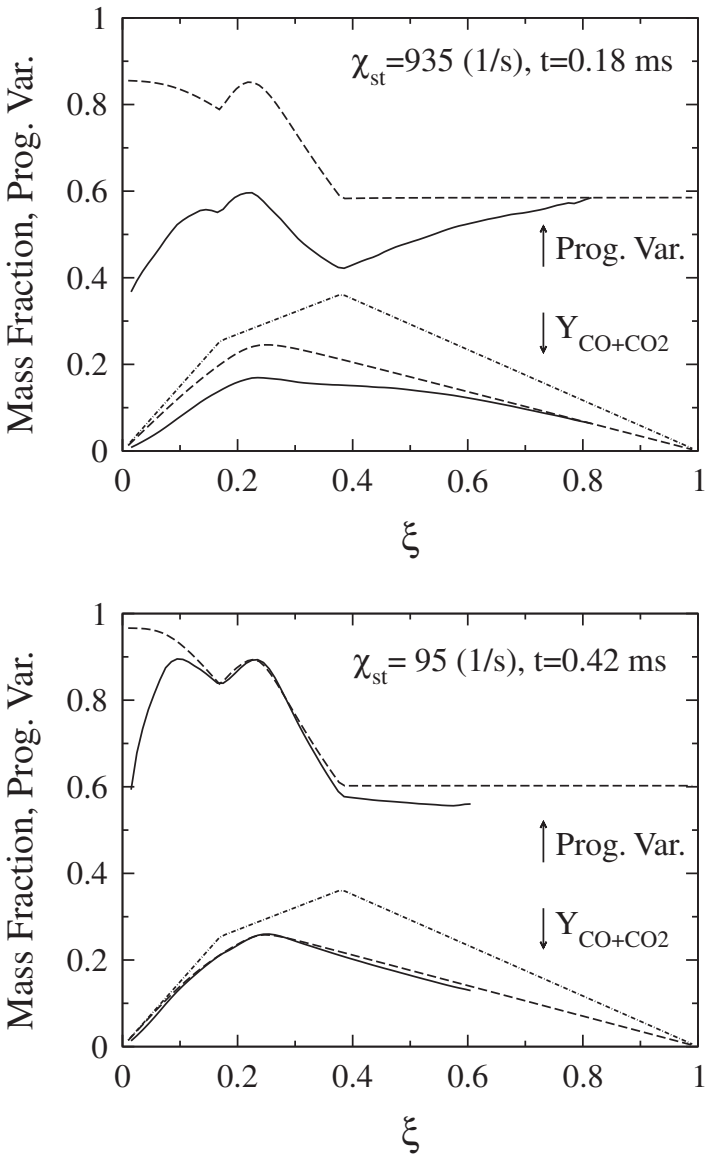

Fig. 15. Progress variable and $\mathrm{CO}+\mathrm{CO}_{2}$ mass fractions for Case 2 at two times. DNS (solid) and steady laminar flamelet (dashed) curves are shown. $Y^{*}$ (dash-dot) is shown for reference.

mass fraction. The higher scalar dissipation value is at the time of the peak flame extinction, and the lower value is at the end of the simulation when the turbulence is relaxing, scalar dissipation decreasing, and the flames are largely reignited. At the later time shown in the figure, the DNS and flamelet results are in good agreement, while, as expected, the DNS and flamelet results differ widely at the time of peak extinction. In both cases, the progress variable is significantly below unity, indicating the flames are far from equilibrium. The shape of the progress variable profiles is similar when $c$ is scaled with equilibrium products instead of ideal combustion products, but the initial dip in the progress variable, while present, is somewhat smoother when using equilibrium products.

The conditional mean progress variable for the three DNS cases is shown in Fig. 16 at several times during the simulations. In each case the progress variable begins at a high value, decreases through the peak extinction time (center time shown for each case), then rises again through the reignition process. Outside the reaction zone, the progress variable is relatively flat, then decreases as extinction and mixing occur. As the flame reignites, these regions gradually begin to recover due to mixing from the reaction zone, but this process is incomplete. The low progress variable at $\xi>\xi_{s t}^{*}$ is due to a much higher concentration of $\mathrm{C}_{2} \mathrm{H}_{4}$ (at the expense of $\mathrm{CO}$ and $\mathrm{CO}_{2}$ ) than occurs at equilibrium.

The profiles of the conditional mean progress variable include intermittent effects (such as burning and extinguished regions). Low values of progress variable may be more reflective of intermittency than a diminished approach to equilibrium in burning re-
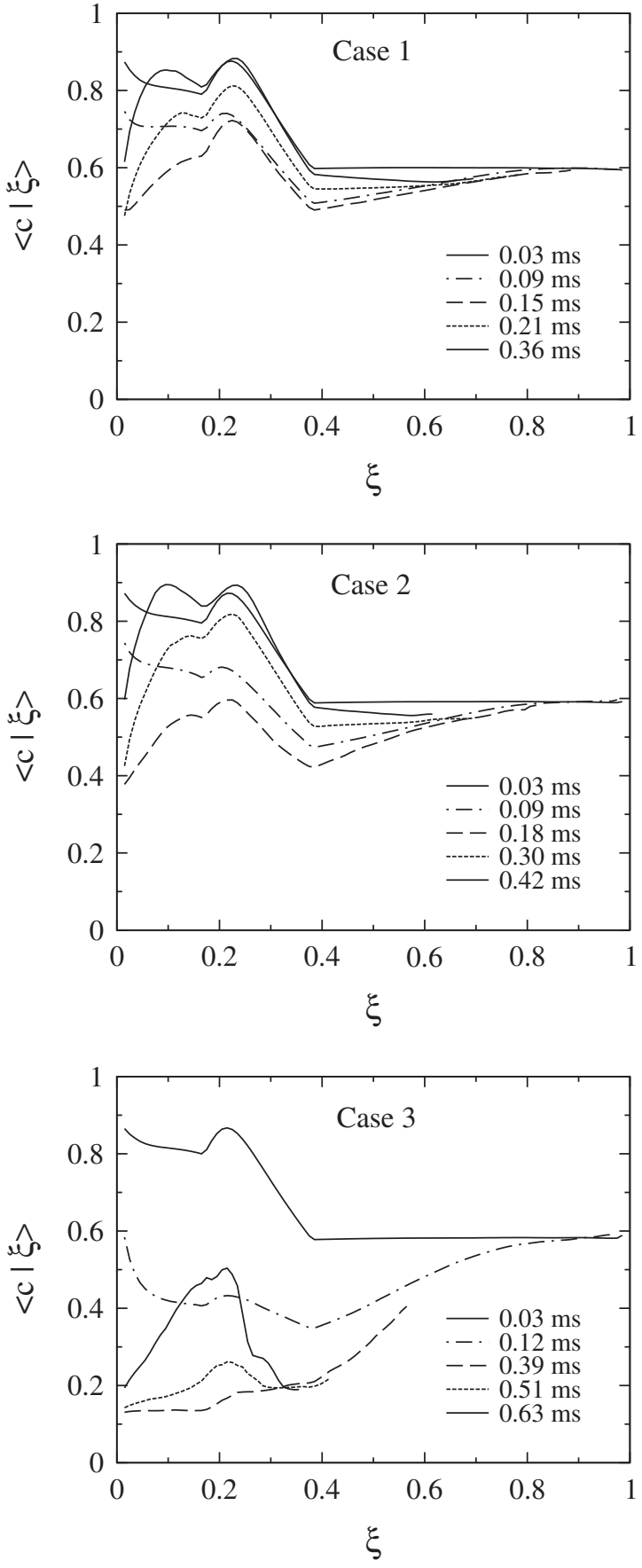

Fig. 16. Progress variable for the three DNS cases at various times.

gions. These effects are not present, however, at the early times due to the initial condition, or at the later times (for Cases 1 and 2 ) as shown by the close agreement between the DNS and flamelet profiles in Fig. 15 at the lower dissipation rate. The progress variable is significantly below unity (especially in rich regions) at both these early and late times.

For Case 3, the lowest value of the progress variable occurs at the time of peak extinction, and occurs near the stoichiometric point, while the progress variable is much higher in regions of higher mixture fraction at that time. This is due to the high degree of extinction and the excess oxidizer. Larger values of mixture fractions consist of a fuel/product mixture, but under fuel-limited conditions, whereas stoichiometric and lean regions are continually mixed with fresh, unreacted oxidizer. 

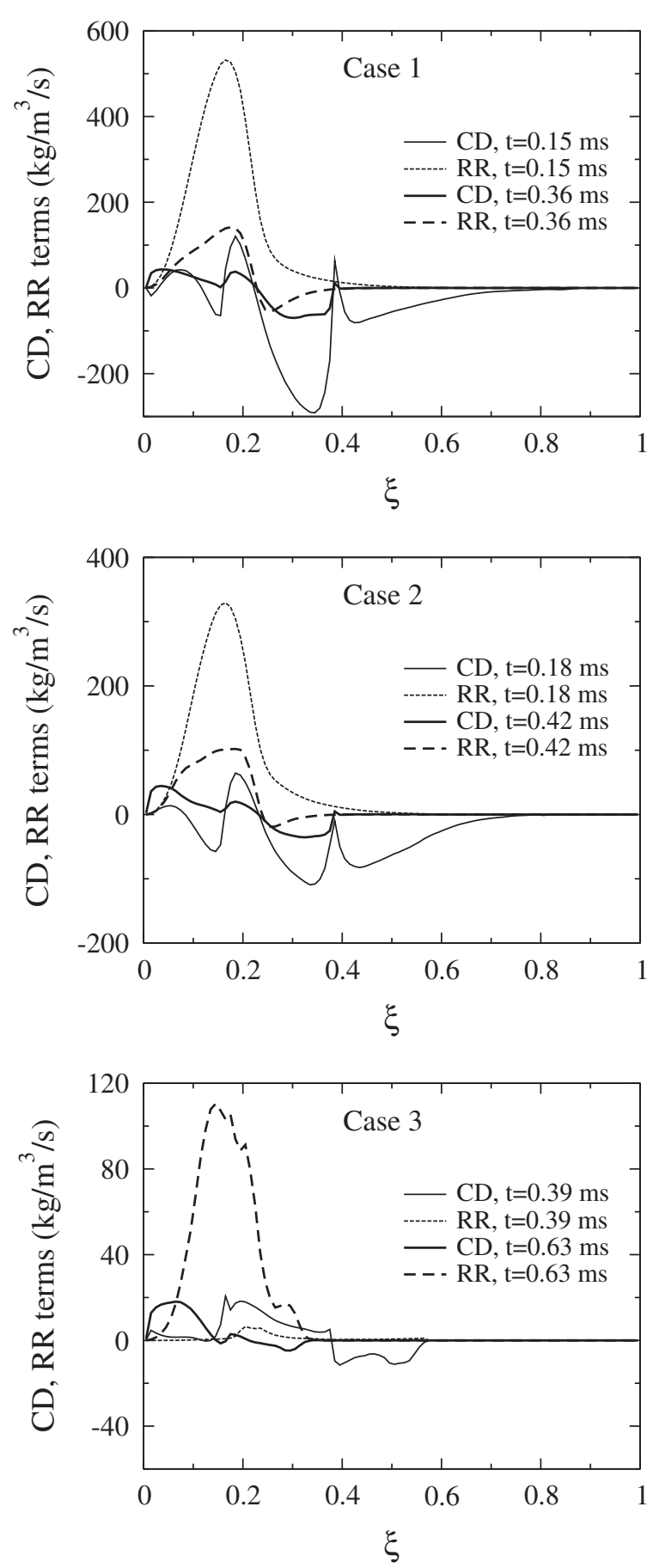

Fig. 17. Plots of the cross dissipation and reaction terms in the progress variable transport equation. Two times are shown for each case, the point of peak extinction and a final recovery time at flame recovery.

A comparison of the conditional mean reaction rate (RR) and cross dissipation (CD) terms in Eq. (13) is shown in Fig. 17. Two times are shown for each of the three cases: the time of peak extinction and end of the simulation at flame recovery. Cases 1 and 2 show similar behavior. The RR and CD terms are higher at the earlier times where mixing and hence burning rates are most intense. The reaction term is active only below a mixture fraction of 0.25 , peaking at the stoichiometric mixture fraction of 0.17 . At both times shown, the RR term is dominant below mixture fractions of 0.25 , whereas the $C D$ term exhibits several peaks and valleys with a strong negative peak at a mixture fraction of 0.35 . The magnitude of the RR and CD terms are higher in Case 1 than in Case 2 owing to the higher Damköhler number and earlier simulation times reported for Case 1 . In Case 3, the reaction rate term at the early time is very small as the flame is nearly extinguished. The peak at $t=0.63 \mathrm{~ms}$ is similar to that of Cases 1 and 2, but at a significantly lower magnitude. In addition, the difference between the $\mathrm{RR}$ and $\mathrm{CD}$ term at the final time for Case 3 is greater than that for Cases 1 and 2 as the flow field is more homogeneous in Case 3 owing to the greater mixing between the fuel and oxidizer streams and products during reignition for Case 3 . This suggests a dominance of reaction over cross dissipation in the progress variable for partially premixed combustion proper in which predominantly premixed flames are propagating in a vitiated flow. In contrast, while the RR term is greater than the $C D$ term in the primarily non-premixed flames, both terms, $R R$ and $C D$, are significant. These results are in agreement with assertions in $[13,28]$.

\section{Conclusions}

A series of three parametric direct numerical simulations of turbulent planar ethylene jet flames has been performed at three values of the Damköhler number at a fixed Reynolds number. These simulations exhibit high levels of flame extinction followed by reignition. The level of extinction ranged between $40 \%$ and nearly $100 \%$. It is shown that, for fixed flow and geometric parameters, the degree of extinction has a significant effect on the development of the flow including the scalar dissipation rate, stoichiometric surface area, and heat release rate evolution. Competition between flame surface area and scalar dissipation rate for overall heat release is shown. Flame extinction is correlated with the sign of curvature of the stoichiometric mixture fraction isosurface, with extinction occurring preferentially in regions of the stoichiometric surface with center of curvature in the fuel stream. The flame reignition mode is examined in terms of a cumulative heat releaseweighted PDF of the flame index, which is used to delineate between non-premixed and premixed flames. The two cases that exhibit smaller degrees of extinction, Case 1, and Case 2, exist predominantly as non-premixed flames through reignition, whereas the reignition mode of Case 3 is dominated by premixed flame propagation as a result of the high degree of extinction followed by mixing of fuel, oxidizer, and combustion products prior to reignition. Comparison of a progress variable iso-surface displacement speed to laminar premixed flame speeds confirmed the predominance of reignition through premixed flame propagation in Case 3. In Case 3, reignition commences fuel-rich of stoichiometric and migrates towards leaner values with peak heat release rates favoring fuel-rich conditions at all times for all three cases. A progress variable based upon $\mathrm{CO}+\mathrm{CO}_{2}$ was defined and analysed. The behavior of this progress variable in time is consistent with the extinction and reignition phenomena. Combustion processes do not approach equilibrium here, resulting in relatively low magnitudes of the progress variable, which is compared between equilibrium, flamelets, and DNS. Comparison of cross dissipation and reaction source terms in the progress variable transport equation shows dominance of the reaction source term. The cross dissipation term is small for Case 3 and more important in Cases 1 and 2 .

\section{Acknowledgments}

Simulations presented in this work were supported by the US Department of Energy, Office of Basic Energy Sciences, Division of Chemical Sciences, Geosciences, and Biosciences. Simulations were performed on the Cray XT4 Jaguar supercomputer at Oak Ridge National Laboratories. 


\section{References}

[1] Y.B. Zeldovich, Combust. Flame 39 (1980) 211-214

[2] J.H. Chen, E.R. Hawkes, R. Sankaran, S. Mason, H.G. Im, Combust. Flame 145 (2006) 128-144.

[3] C. Pantano, J. Fluid Mech. 514 (2004) 231-270.

[4] J. Buckmaster, Prog. Energy Combust. Sci. 28 (2002) 435-475.

[5] E.R. Hawkes, R. Sankaran, J.H. Chen, Extinction and reignition in direct numerical simulations of $\mathrm{CO} / \mathrm{H}_{2}$ temporal plane jet flames, in: 5 th Joint US Combustion Meeting of the Combustion Institute, 2007, p. B05.

[6] S.A. Kaiser, J.H. Frank, Spatial scales of extinction and dissipation in the near field of nonpremixed turbulent jet flames, in: Proceedings of the Combustion Institute, p. 32.

[7] M.R. Overholt, S.B. Pope, Combust. Theory Modell. 3 (1999) 371-408.

[8] G.K.P. Sripakagorn, J. Riley, Combust. Flame 136 (2004) 351-363.

[9] G.K.S. Mitarai, J.J. Riley, Combust. Flame 137 (2004) 306-319.

[10] J.C. Sutherland, P.J. Smith, J.H. Chen, Combust. Theory Modell. 11 (2007) 287303.

[11] E. Hawkes, R. Sankaran, J. Sutherland, J. Chen, Proc. Combust. Inst. 31 (2007) $1633-1640$.

[12] A.R. Masri, R.W. Bilger, Combust. Flame 81 (1990) 260-276

[13] K. Bray, P. Domingo, L. Vervisch, Combust. Flame 141 (2005) 431-437.

[14] J.H. Chen, A. Choudhary, B. de Supinski, M. DeVries, E.R. Hawkes, S. Klasky, W.K. Liao, K.L. Ma, J. Mellor-Crummey, N. Podhorszki, R. Sankaran, S. Shende, C.S. Yoo, Comput. Sci. Discovery 2 (2009) 1-31.
[15] R.J. Kee, G. Dixon-Lewis, J. Warnatz, M.E. Coltrin, J.A. Miller, H.K. Moffat, Transport, Reaction Design, Inc., San Diego, CA, 2000.

[16] C.A. Kennedy, M.H. Carpenter, R.M. Lewis, Appl. Numer. Math. 35 (3) (2000) $177-219$

[17] C.A. Kennedy, M.H. Carpenter, Appl. Numer. Math. 14 (4) (1994) 397-433.

[18] J.C. Sutherland, C.A. Kennedy, J. Comput. Phys. 191 (2003) 502-524.

[19] D.O. Lignell, J.H. Chen, P.J. Smith, T. Lu, C.K. Law, Combust. Flame $151(1-2)$ (2007) 2-28.

[20] Z. Qin, V.V. Lissianski, H. Yang, W.C. Gardiner, S.G. Davis, H. Wang, Proc. Combust. Inst. 28 (2000) 1663-1669.

[21] D.O. Lignell, J.H. Chen, P.J. Smith, Combust. Flame 155 (2008) 316-333.

[22] J. Du, R.L. Axelbaum, Combust. Flame 100 (1995) 367-375.

[23] C. Pantano, S. Sarkar, F.A. Williams, J. Fluid Mech. 481 (2003) 291-328.

[24] L.K. Su, N.T. Clemens, J. Fluid Mech. 488 (2003) 1-29.

[25] H. Yamashita, M. Shimada, T. Takeno, Proc. Combust. Inst. 26 (1996) 27-34.

[26] R. Hilbert, F. Tap, H. el Rabii, D. Thevenin, Progr. Energy Combust. Sci. 30 (2004) 61-117.

[27] R. Kee, J. Grcar, M. Smooke, J. Miller, A fortran program for modeling steady laminar one-dimensional premixed flames, Tech. Rep. SAND85-8240, Sandia National Laboratories, 1985.

[28] P. Domingo, L. Vervisch, K. Bray, Combust. Theory Modell. 6 (2002) 529-551.

[29] P. Domingo, L. Vervisch, D. Veynante, Combust. Flame 152 (2008) 415-432. 